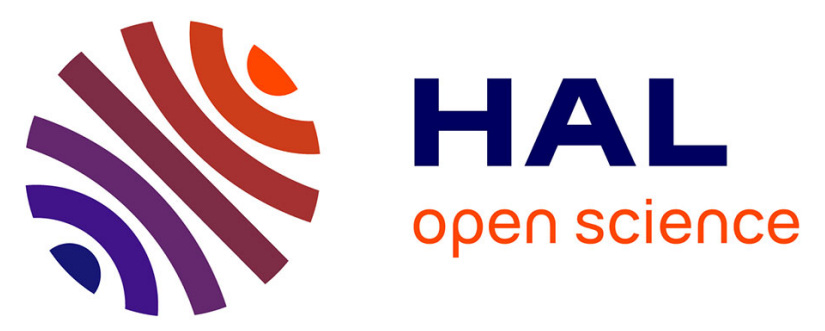

\title{
Illustrations of multi-disciplinary high-fidelity analysis capabilities for aero-structural and aero-acoustic aircraft design
}

Gérald Carrier, Christophe Blondeau, Frédéric Moëns, Marco Carini, Jérémy Cadillon, Thomas Le Garrec, Daniel Mincu

\section{To cite this version:}

Gérald Carrier, Christophe Blondeau, Frédéric Moëns, Marco Carini, Jérémy Cadillon, et al.. Illustrations of multi-disciplinary high-fidelity analysis capabilities for aero-structural and aeroacoustic aircraft design. AIAA Scitech 2021 Forum, Jan 2021, VIRTUAL EVENT, United States. 10.2514/6.2021-1316 . hal-03224254

\section{HAL Id: hal-03224254 \\ https://hal.science/hal-03224254}

Submitted on 11 May 2021

HAL is a multi-disciplinary open access archive for the deposit and dissemination of scientific research documents, whether they are published or not. The documents may come from teaching and research institutions in France or abroad, or from public or private research centers.
L'archive ouverte pluridisciplinaire $\mathbf{H A L}$, est destinée au dépôt et à la diffusion de documents scientifiques de niveau recherche, publiés ou non, émanant des établissements d'enseignement et de recherche français ou étrangers, des laboratoires publics ou privés. 


\title{
Illustrations of multi-disciplinary high-fidelity analysis capabilities for aero-structural and aero-acoustic aircraft design
}

\author{
Gérald Carrier ${ }^{l}$, Christophe Blondeau ${ }^{2}$, Frédéric Moëns ${ }^{3}$, \\ Marco Carini ${ }^{4}$, Jérémy Cadillon ${ }^{5}$, Thomas Le Garrec ${ }^{6}$ and Daniel Mincu ${ }^{7}$ \\ DAAA, ONERA, Paris Saclay University, F-91123, Palaiseau, France
}

This paper presents recent applications of multi-disciplinary optimizations involving high-fidelity analyses for the aerodynamics, structures and aeroacoustics discipline. Three application cases pertaining to aircraft flight physics design are used to illustrate the recent MDAO capabilities developed internally at ONERA.

\section{Nomenclature}

$\begin{array}{ll}\text { BLISS } & =\text { Bi-Level Integrated System Synthesis } \\ \text { CAA } & =\text { Computational Aeroacoustics } \\ \text { CFD } & =\text { Computational Fluid Dynamics } \\ \text { CSM } & =\text { Computational Structural Mechanics } \\ \text { FBO } & =\text { Fan Blade Off } \\ \text { MDAO } & =\text { Multi-Disciplinary Analysis and Optimization } \\ \text { SCTA } & =\text { Supersonic Civil Transport Aircraft } \\ \text { SFC } & =\text { Specific Fuel Consumption }\end{array}$

\section{I.Introduction}

The use of numerical simulations capabilities in the different disciplines involved in the design of an aircraft has massively increased since the emergence in the eighties. For each of these disciplines involved in the aircraft design process, high-fidelity disciplinary analyses capabilities have been deployed in industry design offices and their actual impact on the design processes has increased as their maturity, level of validation and accessible computing resources increased. CFD (Computational Fluid Dynamics), CSM (Computational Structural Modeling) and CAA (Computational Aero-Acoustics) are probably the three types of high-fidelity simulations that are today the most utilized during the design of an aircraft.

\footnotetext{
${ }^{1}$ Research scientist, Aerodynamics Aeroelasticity Acoustics Dept., ONERA, Member AIAA, Gerald.Carrier@onera.fr.

${ }^{2}$ Research scientist, Aerodynamics Aeroelasticity Acoustics Dept., ONERA.

${ }^{3}$ Research scientist, Aerodynamics Aeroelasticity Acoustics Dept., ONERA, Member AIAA.

${ }^{4}$ Research scientist, Aerodynamics Aeroelasticity Acoustics Dept., ONERA, Member AIAA.

${ }^{5}$ Research scientist, Aerodynamics Aeroelasticity Acoustics Dept., ONERA.

${ }^{6}$ Research scientist, Aerodynamics Aeroelasticity Acoustics Dept., ONERA.

${ }^{7}$ Formerly research scientist, Aerodynamics Aeroelasticity Acoustics Dept., ONERA.
} 
With the level of accuracy of these disciplinary simulations capabilities increasing, accounting for the possible couplings between disciplines has become necessary, as the disciplinary level modeling errors become smaller than the error resulting from neglecting those coupling. A good illustration is the need to account explicitly for the aeroelastic deformations of the lifting surfaces of an aircraft when performing aerodynamic performances analysis that appeared when numerical and physical modeling errors of CFD simulations became lower than the error in the geometry resulting from neglecting flexible effects. Another illustration of the need to account for interdisciplinary interactions in order to improve one discipline accuracy is the influence of the inhomogeneous mean aerodynamic field on the acoustic radiation of noise sources. While multi-disciplinary couplings are inherently accounted for during the conceptual design stage using simple (often empirical or semi-empirical) low-fidelity simulations and models, extending such multi-disciplinary simulation capabilities to high-fidelity analyses has been, and still is, a major challenge and active field of research and development.

ONERA has been developing high-fidelity and multi-fidelity MDAO capabilities for at least two decades, essentially for aero-structural design at the earliest stages [1][2]. This paper illustrates the present status of multi-disciplinary simulations capabilities based on high-fidelity numerical methods at ONERA and how they can be applied to practical aircraft design problems. The first section focuses on aero-structural analyses based on high fidelity CFD and CSM methods and illustrate it with the design of the engine integration for a transonic transport aircraft configuration. Then the second section gives an illustration of an aero-acoustic design approach that utilizes both high fidelity CFD and CAA methods to identify optimal tradeoffs between aerodynamic performance and noise signature of a simplified high-lift system. Finally, the last section gives a prospect on ongoing design activity based on CFD and acoustic ray propagation methods to tradeoff between the cruise aerodynamic performances and the sonic boom signature for a low-boom Supersonic Civil Transport Aircraft (SCTA)

\section{II.Aero-structural simulations and associated coupled-sensitivity analysis}

ONERA has been developing for more than fifteen years an aero-structural simulation capability on top of the elsA [3] CFD solver, (Airbus-Safran-ONERA property). This capability is based on a modular, iterative, weak coupling between the fluid solver and a structural solver that can be either a stand-alone, segregated CSM solver (such as NASTRAN), or a reduced-order model of a complex Finite Element Model (FEM) identified a priori and embedded into the CFD simulation environment through a flexibility matrix, or modal projection. This approach is implemented in the elsA-AEL framework (AEL stands for aeroelasticity) that is built on top of the $e l s A$ solver, benefiting from its object-oriented architecture.

Utilizing this segregated partitioned coupling strategy between the fluid and structural solvers, an adjointbased aero-structural sensitivity analysis capability has been developed on top of the aerodynamic adjoint module elsA-Opt [4], to efficiently compute the sensitivity of the coupled system with respect to the aerodynamic shape geometry, and more recently with respect to the structural physical parameters [5].

Such aeroelastic and coupled-adjoint sensitivity analysis capabilities have already been applied to multiple realistic aircraft configurations including multi-point aeroelastic and aerostructural wing design. Indeed flexibility effects must be considered during the cruise segment with adapted lift coefficient, accounting for the wing deformation at the different flight conditions. In this section the theoretical background of the coupled-adjoint equations is first recalled. We then illustrate a typical coupled-adjoint analysis on the Airbus XRF1 aeroelastic configuration. The next sub-section is dedicated to the geometrical parametrization of the CFD and CSM grids with examples for morphing deformation and also for more advanced parametric CAD-based deformation. The last part gives an overview of several projects dedicated to realistic optimization studies during the last decade. 


\section{A. Coupled-adjoint theoretical background}

In order to perform an aero-structural optimization we have to the compute the coupled-adjoint sensitivities with respect to shape and structural design parameters. We refer to structural design parameters when only physical properties of the finite element model are considered, i.e. not affecting the geometry of the analysis model (or at least these geometrical changes are assumed negligible in terms of stiffness variation).

To this end, we need to define the so-called reference shape that will be optimized. In an aeroelastic process, we can choose to optimize a flight shape (typically corresponding to cruise flight conditions) or to optimize an unloaded shape like the jig shape. The aerodynamic jig shape is a natural choice in case of a multi-point optimization study, however this choice is not mandatory. In many situations only the flight shape is at hand from the aircraft manufacturer. Hereafter we recall some theoretical background about the coupled-adjoint sensitivity analysis as it is formulated in the elsA CFD code. For further details, the interested reader can refer to [5][6]. The figure below shows the equilibrium steady configuration and a reference fluid grid aligned with the jig shape. In this specific case, the structural grid is aligned with the reference shape since the structural model should always correspond to the jig shape in its natural (or unloaded) configuration.

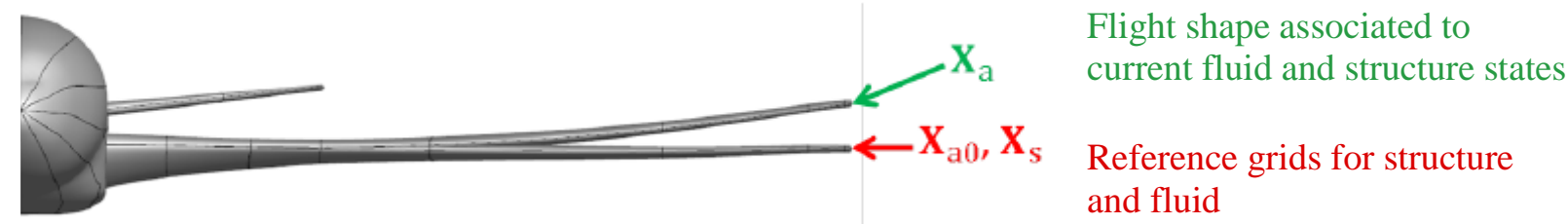

Figure II-1: Definition of aeroelastic equilibrium mesh and parametric reference mesh.

In the following we call the parametric shape $\mathbf{X}_{a}^{0}$ and define it as the reference shape. The structural mesh is noted $\mathbf{X}_{s}$. At the outcome of an aeroelastic analysis, the flow solution $\mathbf{W}$ and the mesh position $\mathbf{X}_{a}$ are available. The steady aeroelastic analysis is an iterative process and at each fluid-structure coupling a mesh deformation is applied to the fluid domain. This process is illustrated in Figure II-2.

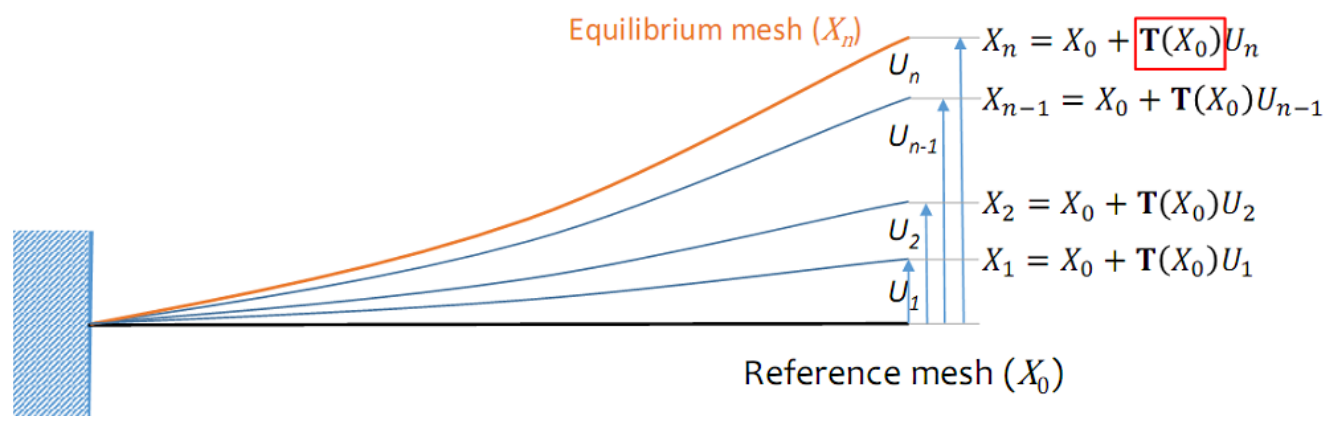

Figure II-2: Iterative mesh deformation strategy based on a reference mesh.

It is worth mentioning that in the proposed approach the mesh deformation operator merely depends on the reference grid $\mathbf{X}_{a}^{0}$ and is supposed to be robust enough to absorb the potential large structural displacements. The aerodynamic volume displacement field $\delta \mathbf{X}_{a}$ is always defined relative to the aerodynamic reference mesh $\mathbf{X}_{a}^{0}$ and the structural displacements $\mathbf{U}$ relative to the structural mesh $\mathbf{X}_{s}$. We then have 


$$
\mathbf{X}_{a}=\mathbf{X}_{a 0}+\delta \mathbf{X}_{a}\left(\delta \mathbf{X}_{a, \text { surf }}, \mathbf{X}_{a}^{0}\right)=\mathbf{X}_{a}^{0}+\mathbf{T}_{\text {vol }}\left(\mathbf{X}_{a}^{0}\right) \delta \mathbf{X}_{a, \text { surf }}
$$

where $\delta \mathbf{X}_{a, \text { surf }}$ denotes the aerodynamic displacement field at the aeroelastic interface (wall boundary). The mesh deformation operator is called $\mathbf{T}_{\text {vol }}$. The displacement field $\delta \mathbf{X}_{a, \text { surf }}$ is computed by smoothing the structural displacements $\mathbf{U}$ on the aerodynamic surface grid using the linear operator $\mathbf{T}_{\text {surf }}^{U}$.

$$
\delta \mathbf{X}_{a, \text { surf }}=\delta \mathbf{X}_{a, \text { surf }}\left(\mathbf{X}_{a}^{0}, \mathbf{X}_{s}, \mathbf{U}\right)=\mathbf{T}_{\text {surf }}^{U}\left(\mathbf{X}_{a}^{0}, \mathbf{X}_{s}\right) \mathbf{U}
$$

The aerodynamic loads $\mathbf{Q}_{a}$ are transferred onto the structural mesh using the linear load transfer operator $\mathbf{T}_{\text {surf }}^{Q}$ to give the structural loads $\mathbf{Q}_{s}$ in the following form:

$$
\mathbf{Q}_{s}\left(\mathbf{Q}_{a}\left(\mathbf{W}, \mathbf{X}_{a}\right), \mathbf{X}_{a}^{0}, \mathbf{X}_{s}\right)=\mathbf{T}_{\text {surf }}^{Q}\left(\mathbf{X}_{a}^{0}, \mathbf{X}_{s}\right) \mathbf{Q}_{a}\left(\mathbf{W}, \mathbf{X}_{a}\right)
$$

The discrete structural and aerodynamic residuals are then linearized around the aeroelastic equilibrium, and after some algebra manipulation, the coupled linear system defining the adjoint vectors $\lambda_{a}$ and $\lambda_{s}$ is written as:

$$
\left[\begin{array}{ll}
{\left[\frac{\partial \mathbf{R}_{a}}{\partial \mathbf{W}}\right]^{T}} & -[\mathbf{C}]^{T} \\
{[\mathbf{A}]^{T}\left[\frac{\partial \mathbf{R}_{a}}{\partial \mathbf{X}_{a}}\right]^{T}} & \mathbf{K}^{T}-[\mathbf{D}]^{T}
\end{array}\right]\left(\begin{array}{l}
\lambda_{a} \\
\lambda_{s}
\end{array}\right)=\left(\begin{array}{l}
-\left[\frac{\partial \mathrm{J}}{\partial \mathbf{W}}\right]^{T} \\
-[\mathbf{A}]^{T}\left[\frac{\partial \mathrm{J}}{\partial \mathbf{X}_{a}}\right]^{T}-\left[\frac{\partial \mathrm{J}}{\partial \mathbf{U}}\right]^{T}
\end{array}\right)
$$

where $\mathbf{R}_{a}$ is the fluid discrete residual block matrix, $\mathbf{K}$ the structural stiffness matrix and $\mathrm{J}$ the scalar function of interest like lift, drag or moment coefficient. All matrices $[\mathbf{A}],[\mathbf{B}],[\mathbf{C}]$ and $[\mathbf{D}]$ appearing in this section are analytic and will not be further detailed here (see [6]).

Once the adjoint vectors are computed, the total derivative of the objective function with respect to the design parameters $\mathbf{p}$ is given by

$$
\begin{aligned}
\frac{\mathrm{dJ}}{\mathrm{d} \mathbf{p}} & =\left(\frac{\partial \mathrm{J}}{\partial \mathbf{X}_{a}}+\lambda_{a}^{T} \frac{\partial \mathbf{R}_{a}}{\partial \mathbf{X}_{a}}-\lambda_{s}^{T} \mathbf{S} \mathbf{T}_{\text {surf }}^{Q} \frac{\partial \mathbf{Q}_{a}}{\partial \mathbf{X}_{a}}\right)\left([\mathbf{B}] \frac{\mathrm{d} \mathbf{X}_{a}^{0}}{\mathrm{~d} \mathbf{p}}+[\mathbf{F}] \frac{\mathrm{d} \mathbf{X}_{s}}{\mathrm{~d} \mathbf{p}}\right) \\
& -\lambda_{s}^{T} \mathbf{S}\left(\frac{\partial \mathbf{Q}_{s}}{\partial \mathbf{X}_{a}^{0}} \frac{\mathrm{d} \mathbf{X}_{a}^{0}}{\mathrm{~d} \mathbf{p}}+\frac{\partial \mathbf{Q}_{s}}{\partial \mathbf{X}_{s}} \frac{\mathrm{d} \mathbf{X}_{s}}{\mathrm{~d} \mathbf{p}}\right)-\lambda_{s}^{T} \frac{\partial \mathbf{S}}{\partial \mathbf{p}} \mathbf{Q}_{s}+\frac{\partial \mathrm{J}}{\partial \mathbf{X}_{s}} \frac{\mathrm{d} \mathbf{X}_{s}}{\mathrm{~d} \mathbf{p}}
\end{aligned}
$$

where $\mathbf{S}$ is the structural flexibility matrix. The flexibility matrix $\mathbf{S}$ is a condensed form of the inverse of the stiffness matrix which is built in an external pre-processing step such that $\mathbf{U}=\mathbf{S} \mathbf{Q}_{s}$.

The coupled-adjoint sensitivity analysis module requires as input one reference volume mesh sensitivity $\mathrm{d} \mathbf{X}_{a}^{0} / \mathrm{d} p$ and one structural grid sensitivity $\mathrm{d} \mathbf{X}_{s} / \mathrm{d} p$ for each design variable. Like the parametric reference mesh, the volume term $\mathrm{d} \mathbf{X}_{a}^{0} / \mathrm{d} p$ is obtained by applying a mesh deformation operator such that $\mathrm{d} \mathbf{X}_{a}^{0} / \mathrm{d} p=$ $\left[\mathbf{T}_{\text {vol }}\left(\mathbf{X}_{a}^{a b s}\right)\right] \mathrm{d} \mathbf{X}_{a, \text { surf }}^{0} / \mathrm{d} p$, where the last term corresponds to the wall grid sensitivity. Typical strategies for shape parametrization will be discussed in section $\mathrm{C}$. In the above we have introduced a new grid $\mathbf{X}_{a}^{a b s}$ which is an absolute CFD grid which represents a constant mesh not affected by design parameters. 
In practice this mesh usually coincides with the initial mesh of a shape optimization process. As a consequence, the operator $\mathbf{T}_{v o l}\left(\mathbf{X}_{a}^{a b s}\right)$ is constant.

In order to separate geometric and parametric contributions, the reference mesh sensitivity $\mathrm{d} \mathbf{X}_{a}^{0} / \mathrm{d} \mathbf{p}$ and the structural grid sensitivity $\mathrm{d} \mathbf{X}_{s} / \mathrm{d} p$ can be factored out in Equation (II-5) to give:

$$
\frac{\mathrm{dJ}}{\mathrm{d} \mathbf{p}}=\frac{\partial \mathfrak{J}}{\partial \mathbf{X}_{a}^{0}} \frac{\mathrm{d} \mathbf{X}_{a}^{0}}{\mathrm{~d} \mathbf{p}}+\frac{\partial \mathfrak{J}}{\partial \mathbf{X}_{s}} \frac{\mathrm{d} \mathbf{X}_{s}}{\mathrm{~d} \mathbf{p}}-\lambda_{s}^{T} \frac{\partial \mathbf{S}}{\partial \mathbf{p}} \mathbf{Q}_{s}
$$

The aerodynamic geometric adjoint is then defined as:

$$
\frac{\partial \mathfrak{S}}{\partial \mathbf{X}_{a}^{0}}=\left(\frac{\partial \mathrm{J}}{\partial \mathbf{X}_{a}}+\lambda_{a}^{T} \frac{\partial \mathbf{R}_{a}}{\partial \mathbf{X}_{a}}-\lambda_{s}^{T} \mathbf{S} \mathbf{T}_{\text {surf }}^{Q} \frac{\partial \mathbf{Q}_{a}}{\partial \mathbf{X}_{a}}\right)[\mathbf{B}]-\lambda_{s}^{T} \mathbf{S} \frac{\partial \mathbf{Q}_{s}}{\partial \mathbf{X}_{a}^{0}}
$$

The structural geometric counterpart is given by:

$$
\frac{\partial \widetilde{I}}{\partial \mathbf{X}_{s}}=\left(\frac{\partial \mathrm{J}}{\partial \mathbf{X}_{a}}+\lambda_{a}^{T} \frac{\partial \mathbf{R}_{a}}{\partial \mathbf{X}_{a}}-\lambda_{s}^{T} \mathbf{S} \mathbf{T}_{\text {surf }}^{Q} \frac{\partial \mathbf{Q}_{a}}{\partial \mathbf{X}_{a}}\right)[\mathbf{F}]-\lambda_{s}^{T} \mathbf{S} \frac{\partial \mathbf{Q}_{s}}{\partial \mathbf{X}_{s}}+\frac{\partial \mathrm{J}}{\partial \mathbf{X}_{s}}
$$

This quantity is obtained at the outcome of a coupled-adjoint analysis. The final gradient assembly then only consists in matrix-vector products. Matrix operators $[\mathbf{B}]$ and $[\mathbf{F}]$ embed the geometric derivatives of transfer operators $\mathbf{T}_{\text {surf }}^{U}$ and $\mathbf{T}_{\text {surf }}^{Q}$ and also of the volume mesh deformation operator $\mathbf{T}_{\text {vol }}$. A usual and legitimate practice consists in considering that structural design parameters $\mathbf{p}_{s}$ related to physical properties only do not affect grid coordinates, i.e. $\mathrm{d} \mathbf{X}_{a}^{0} / \mathrm{d} \mathbf{p}_{s}=\mathbf{0}$ and $\mathrm{d} \mathbf{X}_{s} / \mathrm{d} \mathbf{p}_{s}=\mathbf{0}$. The total derivative of the function of interest then greatly simplifies into

$$
\frac{\mathrm{dJ}}{\mathrm{d} \mathbf{p}}=-\lambda_{s}^{T} \frac{\partial \mathbf{S}}{\partial \mathbf{p}} \mathbf{Q}_{s}
$$

Another usual assumption is to assume that the structural stiffness sensitivity with respect to local aerodynamic wall shape changes $\mathbf{p}_{a}$ (typically airfoil sections) is negligible. In this case the total derivative ends up to

$$
\frac{\mathrm{dJ}}{\mathrm{d} \mathbf{p}}=\left(\frac{\partial \mathrm{J}}{\partial \mathbf{X}_{a}}+\lambda_{a}^{T} \frac{\partial \mathbf{R}_{a}}{\partial \mathbf{X}_{a}}-\lambda_{s}^{T} \mathbf{S} \mathbf{T}_{s u r f}^{Q} \frac{\partial \mathbf{Q}_{a}}{\partial \mathbf{X}_{a}}\right)[\mathbf{B}] \frac{\mathrm{d} \mathbf{X}_{a}^{0}}{\mathrm{~d} \mathbf{p}}-\lambda_{s}^{T} \mathbf{S} \frac{\partial \mathbf{Q}_{s}}{\partial \mathbf{X}_{a}^{0}} \frac{\mathrm{d} \mathbf{X}_{a}^{0}}{\mathrm{~d} \mathbf{p}}
$$

The expression above also corresponds to the gradient reconstruction for an aeroelastic optimization with respect to shape design variables only. Finally, when design variables have a global nature (like wing planform sweep or span) all the contributions in Equation (II-5) have to be taken into account.

\section{B. Coupled-adjoint system solution}

A partitioned approach similar to a Linear Block Gauss-Seidel (LBGS) relaxation is used to solve system (II-4). Currently a flexible GMRES solver with deflated restarting (FGMRES-DR) is used for the solution of the system of fluid discrete linearized equations [7]. A nested strategy is adopted where the outer GMRES is pre-conditioned by an inner GMRES which is in turn pre-conditioned by a Block-LUSGS 
relaxation or a Block-ILU0 incomplete factorization applied to a $1^{\text {st }}$ order approximate flux Jacobian matrix. This matrix has a very compact stencil and is then very efficiently factorized. This class of nested GMRES solvers has proven efficient even for stiff problems where the turbulence model is fully linearized [8].

We illustrate the typical convergence behavior of the coupled-adjoint system on the well-known Airbus XRF-1 research model, representative of a large passenger aircraft. The wing-body configuration is retained for the aerodynamic grid while a complete structural model is considered (see Figure II-3). The size of the fluid grid is about 5.3M points. The one-equation Spalart-Allmaras model has been adopted for turbulence modelling. It is worth mentioning that all adjoint sensitivities are computed by considering a fully linearized turbulence model. The coupled-adjoint system is solved with three right-hand sides associated to pressure drag, lift and moment coefficients.
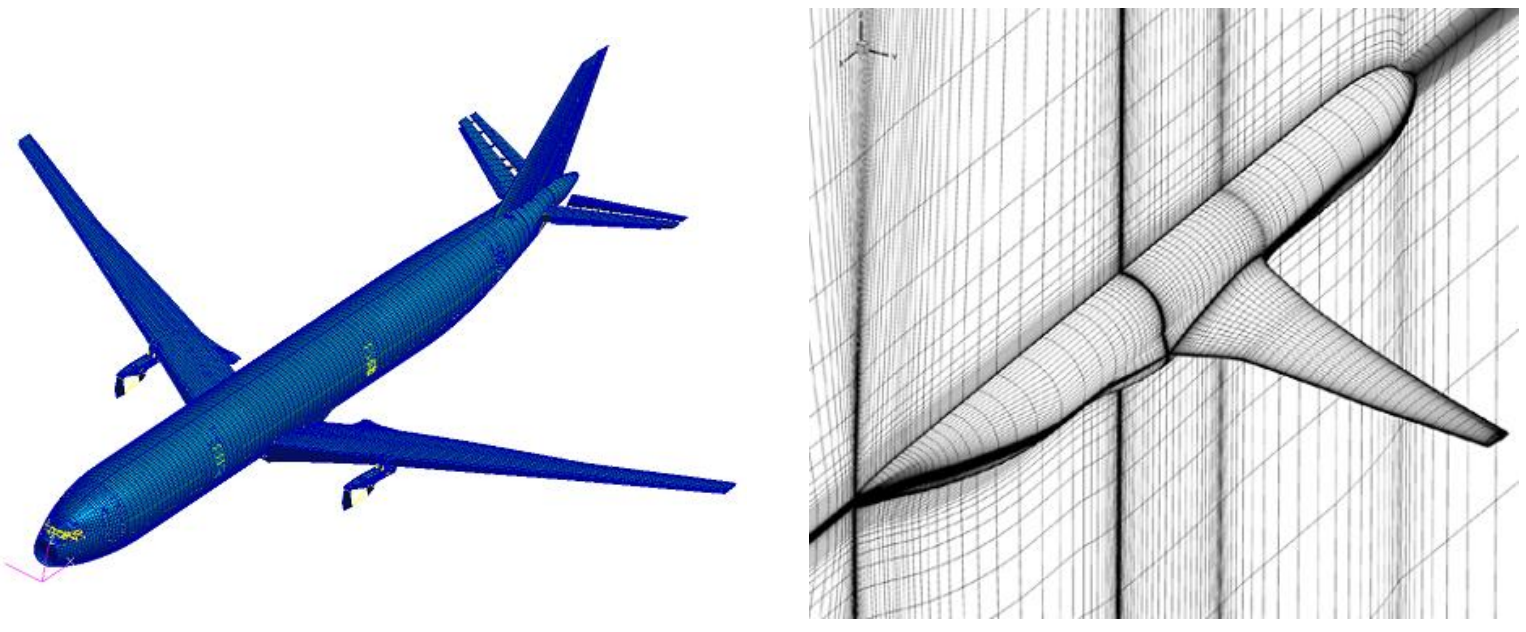

Figure II-3: Structural and aerodynamic meshes for the XRF-1 configuration.

In the left-hand side of Figure II-4 a typical convergence for a rigid aerodynamic adjoint is plotted. The corresponding convergence for the solution of the coupled-adjoint system is given in the right-hand side where each circle marker indicates an aeroelastic coupling in the LBGS solver.

Looking at the convergence plot profile for the coupled-adjoint linear system, we understand how the efficiency of the GMRES solver attached to the block of fluid equations (between two fluid-structure couplings) has a considerable impact on the global efficiency. This is why a lot of time was spent recently in the improvement of the fluid GMRES solver. For the specific case of coupled-adjoint performance improvement, we systematically store the full first order flux Jacobian matrix used for preconditioning. Also for the exact Jacobian matrix-vector product, the invariant part (i.e. terms not depending on the adjoint vector) in the assembly process is stored while the other part is still computed on-the-fly. We also implemented all the intensive linear algebra operations using the BLAS and LAPACK Intel Math Kernel Library (MKL) with multi-threading and vectorization acceleration as much as possible. We then noticed that for the 3D XRF1 test case (coarse mesh of about 5.3M cells split in 690 domains and distributed over 112 CPUs) the acceleration factor was about 3 compared to the original coupled-adjoint implementation designed to be memory efficient with no storage of Jacobian matrix and on-the-fly linear algebra only. This work was partially performed in the context of the EU project MADELEINE (Multidisciplinary 
ADjoint-based Enablers for LargE-scale Industrial desigN in aEronautics) dedicated to the adjoint-based optimization of realistic industrial configurations [9][10].
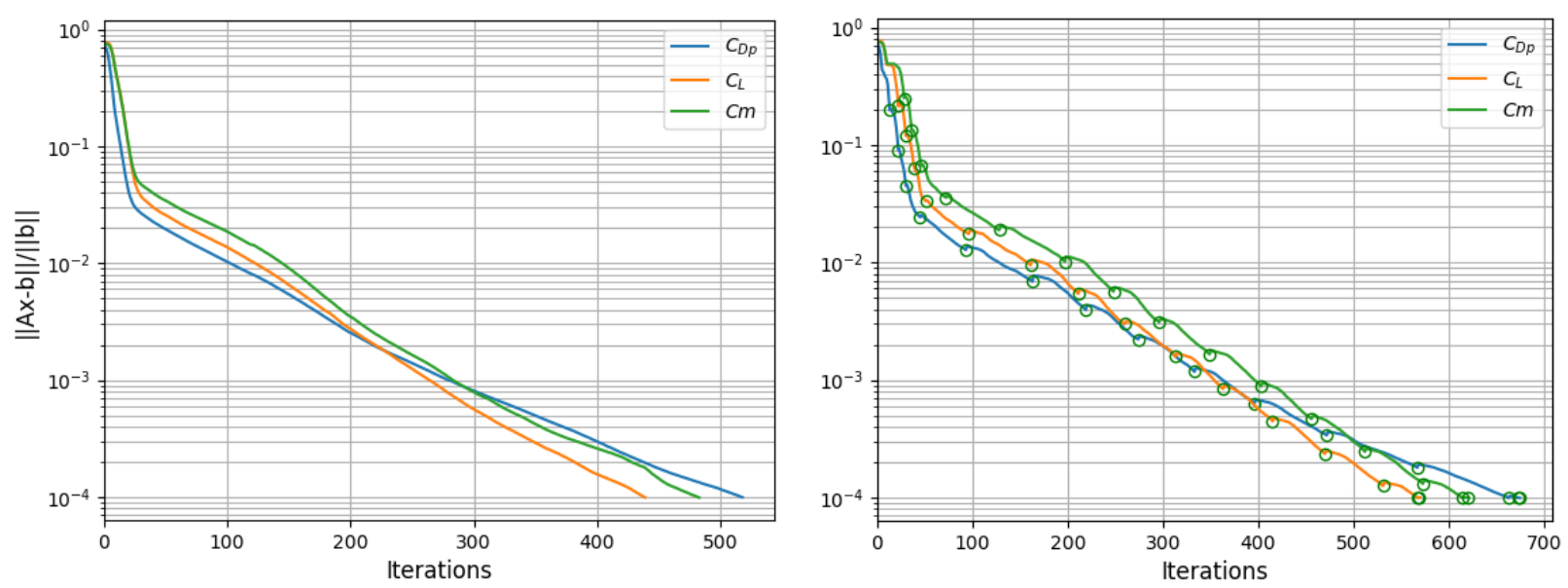

Figure II-4: XRF1 test case - Rigid and coupled-adjoint density residual norm convergence for $\mathrm{C}_{\mathrm{Lp}}, \mathrm{C}_{\mathrm{Dp}}$ and $\mathrm{C}_{\mathrm{M}}$.

\section{Parametrization and mesh deformation}

This section covers various strategies for shape control and parametrization for structural and aerodynamic meshes.

\section{Mesh morphing - free form deformation}

Most of the time for multi-disciplinary optimization studies industrial partners provide frozen datum grids for structural and aerodynamic analyses. This is where morphing tools can be of great help to deform existing meshes. However, even if this approach seems very appealing, achieving a fine control of the shape allowing a manufacturable optimized design may be a great challenge. An example is given hereafter about an aero-structural design of an under-wing engine's pylon which was performed during the EU projects VIVACE (Value Improvement through a Virtual Aeronautical Collaborative Enterprise) [12] and CRESCENDO (Collaborative \& Robust Engineering using Simulation Capability Enabling Next Design Optimization) [13]. The shape of the pylon structural box and aerodynamic fairing was allowed to change in width at several locations and also in height (aircraft vertical axis) and length (aircraft longitudinal axis). The engine pylon is typically a very complex structural part which requires a proprietary industrial knowledge for automatic parametric grid mesh generation and sizing. This knowledge is not supposed to be shared with external partners and an elegant fallback solution is then to consider morphing tools. However, setting up the morphing domains is complex as it requires at some point an automatic feature recognition of the primary structural members like ribs, stiffeners, flanges ... If the morphing domains and the associated kinematic boundary conditions are not properly defined, the primary structural members will eventually experience some nonrealistic geometry variation that may lead to unrealistic structural analysis responses. For instance buckling is very sensible to variations in geometry. An example of variation of the length and height of the pylon structural box is shown in Figure II-5. The free-form deformation strategy is also often used for aerodynamic shape control. An example of directional free-form is given in Figure II-6 for the same pylon. These tools have also to be scriptable in order to be embedded in the optimization framework. In addition, a differentiated version is very 
helpful to obtain the sensitivity of grid coordinates with respect to the shape design parameters. This feature is quite standard for aerodynamic grids for which only wall sensitivities are required. However, for structural meshes the whole set of internal and external grid coordinates has to be differentiated. It is also worth mentioning that realistic structural finite element models consists in many elements of very different types which brings a nontrivial additional complexity to the geometric differentiation process. This is why the current practice is still to obtain derivatives using a parallelized finite difference approximation.
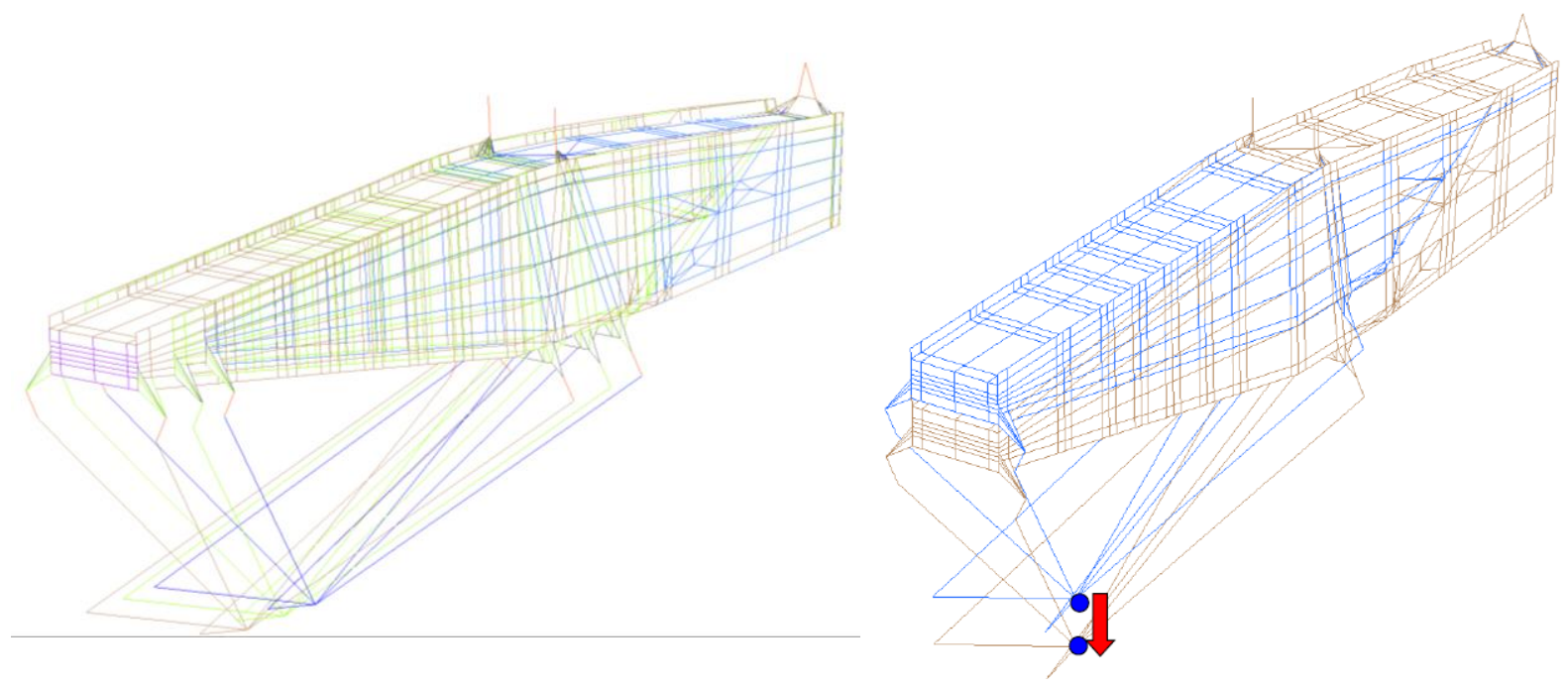

Figure II-5: Example of pylon box morphing for a longitudinal and vertical displacement of the engine central attachment.
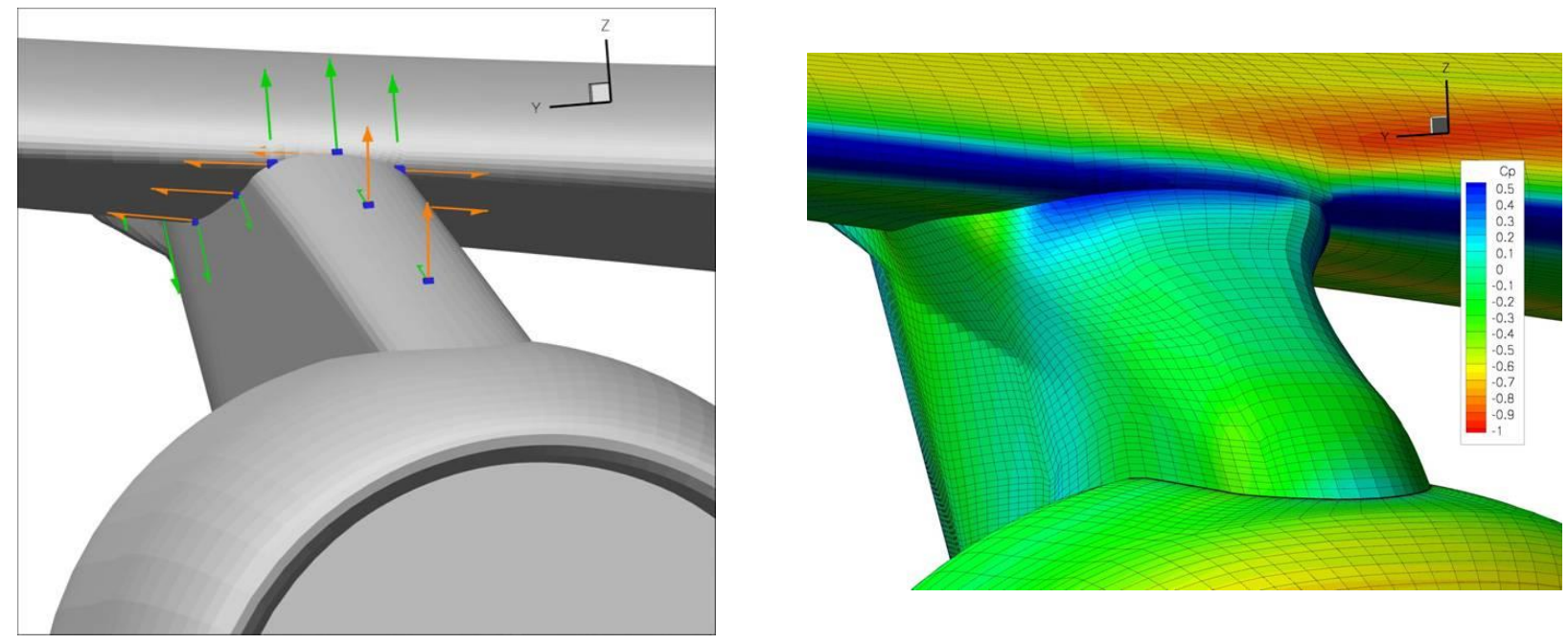

Figure II-6: Example of morphing of the shape of pylon fairing.

Another emblematic application of the morphing approach was performed during the French ARTEMIS project dedicated to bi-level approaches for aero-structural optimization of the Airbus XRF-1 wing [15]. The structural model has already been presented in Figure II-3. The sweep angle, taper ratio, span and height of the wing box were part of the set of design variables. The morphing domain was divided into elementary volumes embedding the primary structural members of the wingbox. A first step of features 
extraction was performed leading to a skeleton CAD of the wing box. The elementary morphing domains were then designed to ensure planar deformations of spars and ribs during the morphing process, resulting in a highly constrained geometric operation. An example of $+/-3^{\circ}$ of sweep variation is presented in Figure II-7. The corresponding sensitivities were obtained using finite differences.

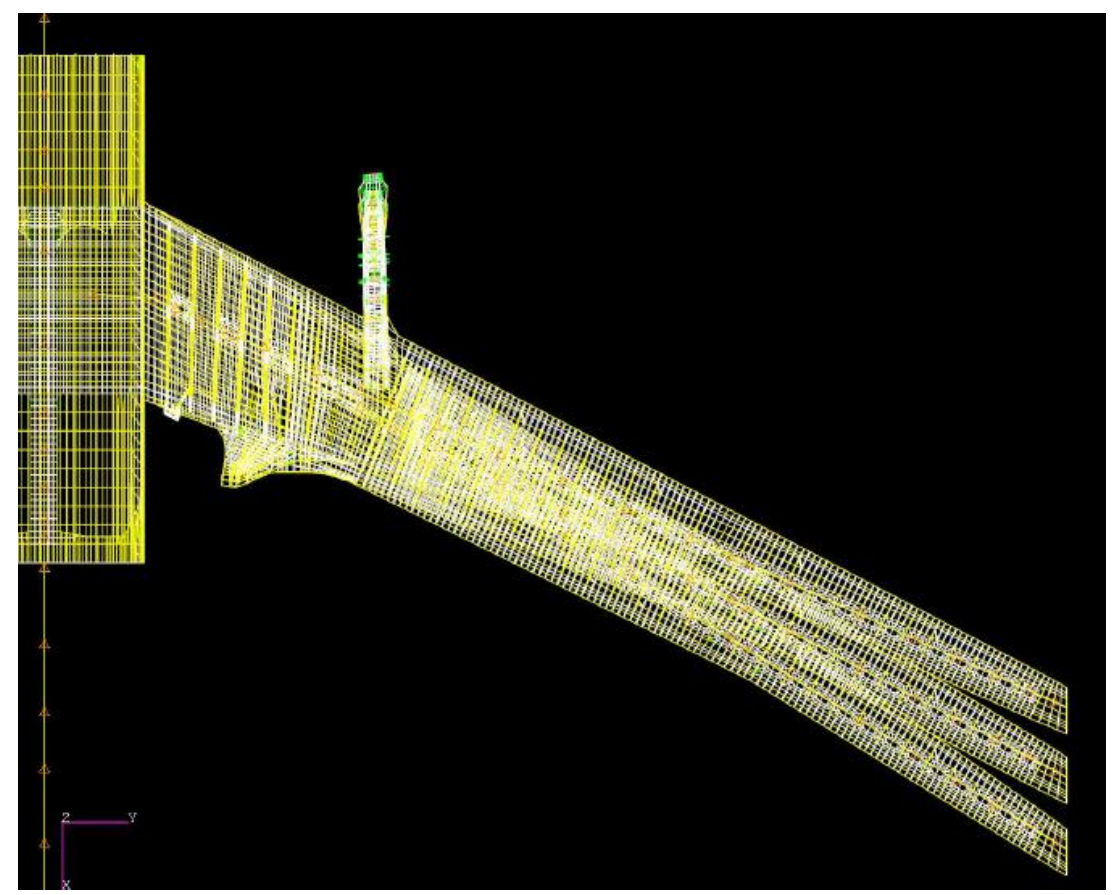

Figure II-7: Airbus XRF-1 configuration, wing box morphing for $+/-3^{\circ}$ of sweep angle.

\section{Parametric cad for mesh deformation and grid sensitivity}

A powerful approach to drive the geometric changes of the aerodynamic grid during an optimization process is to rely on a differentiated parametric CAD model. We illustrate this on a simple engineering parametrization of the XRF-1 wing shape. This work was performed in the context of the EU project MADELEINE. The parametric CAD modeler PADGE (Parametric And Differentiated Geometrical Engine), was kindly provided by Airbus for this task. PADGE is based on NURBS and allows to model aircraft components and to compute shape deformations and their related geometric sensitivities. This approach is particularly suitable in an industrial context as it allows to retrieve a CAD model directly from the optimization process. It also guarantees that the output CAD model is exactly aligned with the surface mesh without necessitating an additional process to either extract a usable geometry, for instance from the deformed surface mesh, or check that the output is actually well-aligned with the optimized deformation. Handling of complex CAD intersections is also a standard feature of the tool.

This tool offers a wide range of interpolation and surfacing methods to build advanced CAD surface models that can then be controlled by a subset of design variables. The use of templates and parameters allows to natively handle critical geometric design constraints such as leading edge radius, trailing edge angles or thickness distribution, thus not necessitating to define explicit constraints that must then be handled by the optimization algorithm. A mesh deformation approach is then used to either propagate the surface mesh deformation to the volume mesh or to compute the mesh adjoint in order to assemble geometric gradients. 
In order to properly instantiate a template, a matching process is first required. More specifically, an inverse least-squares problem is solved to fit the parametric CAD as close as possible onto a given target aerodynamic wall grid. As an example, a simple CAD model has been built based on 4 airfoil sections extracted from the aerodynamic wall grid of the XRF1 wing-body use case (see right-hand side image in Figure II-8). Also, the leading and trailing edge guidelines are extracted from the wall grid and provided to the CAD fitting process to compute the global wing features like twist distribution and airfoil global position. Figure II-9: Figure II-9 shows the PADGE main window where the datum jig and flight shapes are plotted. The red skeleton represents the CAD model after fitting onto the flight configuration. It is seen in the right-hand side of Figure II-9 how well the CAD matches the aerodynamic shape. Once the CAD model internal controller values are initialized, the independent design variables whose values are allowed to change need to be defined.
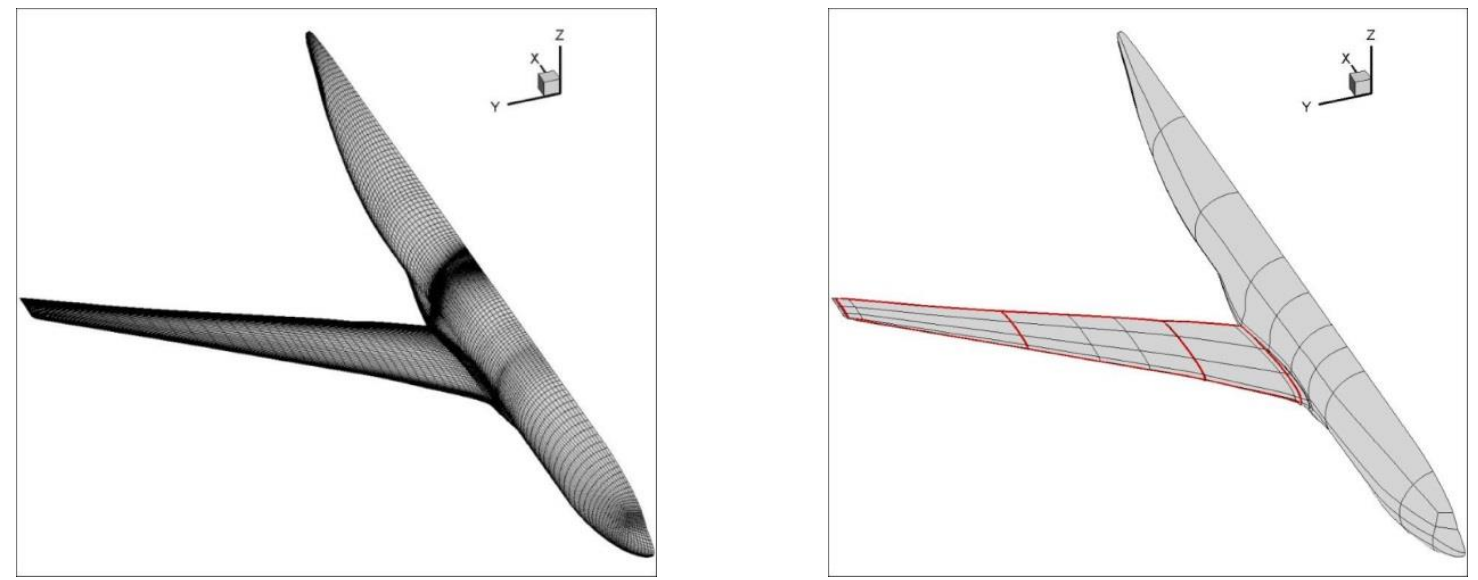

Figure II-8: Matching data extracted from the jig CFD wall grid for the parametric CAD model.
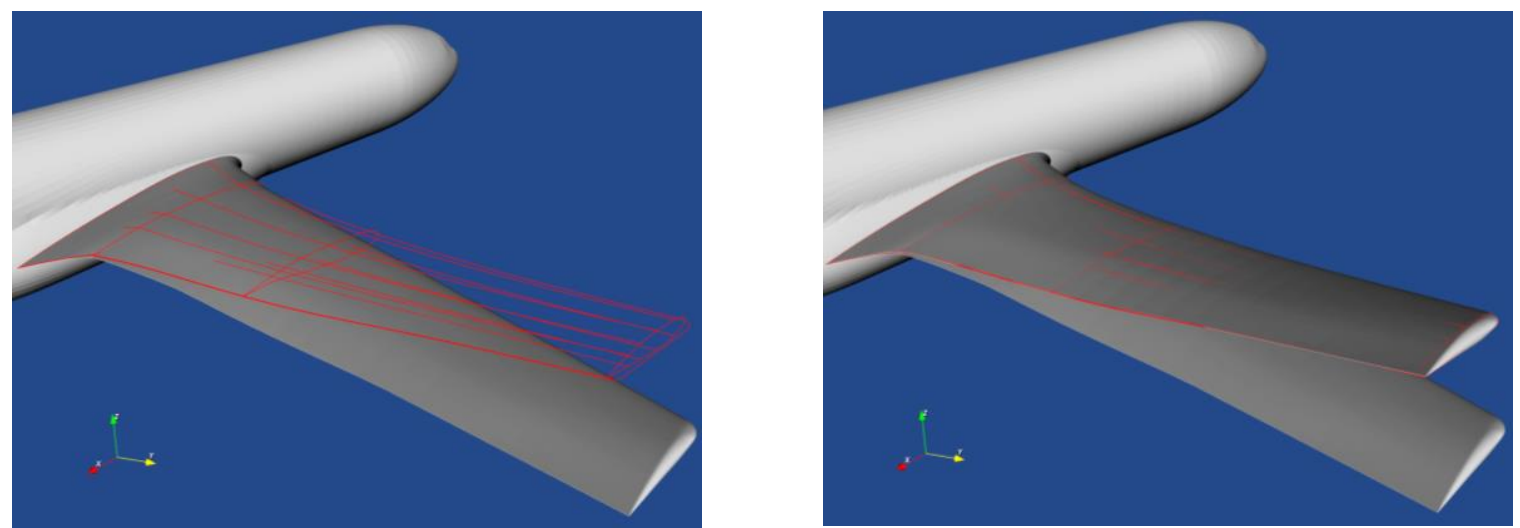

Figure II-9: CAD model fitted to the wing flight shape. 

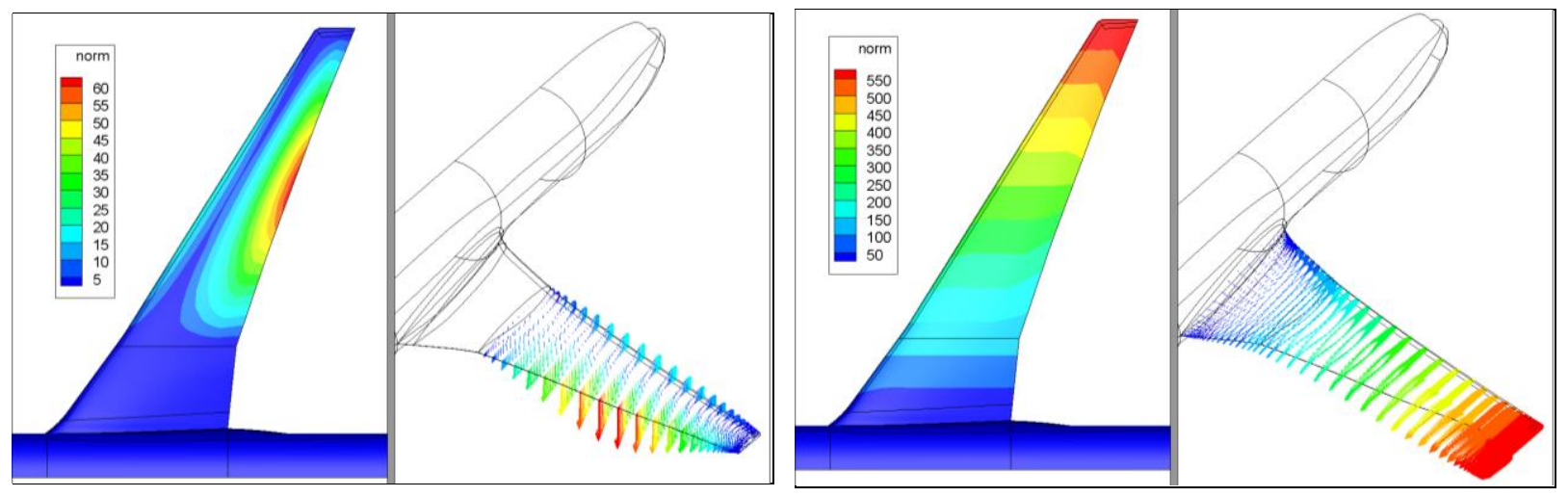

Figure II-10: Sensitivity of wall grid coordinates (colored by vector norm) with respect to a variation of mid-outer wing twist (top) and of sweep angle (bottom).

In section A we defined the so-called reference mesh $\mathbf{X}_{a}^{0}\left(\mathbf{p}_{k}\right)$ which depends on the design parameters. However, the procedure for the obtention of this mesh and its associated sensitivity $\mathrm{d} \mathbf{X}_{a}^{0} / \mathrm{d} \mathbf{p}$ was not detailed. Let us define $\mathbf{X}_{a}^{a b s}$ an absolute CFD grid which represents a constant mesh not affected by design parameters. In practice this mesh usually coincides with the initial mesh of a design optimization process. Let us $\mathbf{p}_{\text {ini }}$ be the set of initial design parameters and $\mathbf{X}_{a}^{0}\left(\mathbf{p}_{k}\right)$ the parametric mesh associated to a set of design variables $\mathbf{p}_{k}$ at iteration $k$, we assume that $\mathbf{X}_{a}^{a b s}=\mathbf{X}_{a}^{0}\left(\mathbf{p}_{\text {ini }}\right)$. Given $\mathbf{p}_{k}$, the parametric mesh generation process starts by creating a displacement field for the absolute mesh wall grids $\delta \mathbf{X}_{a, \text { surf }}\left(\mathbf{X}_{a}^{a b s}, \mathbf{p}_{k}\right)$ which merely depends on the absolute grid coordinates. A mesh deformation process is then applied to obtain the parametric volume mesh $\mathbf{X}_{a}^{0}\left(\mathbf{p}_{k}\right)$ such that $\mathbf{X}_{a}^{0}\left(\mathbf{p}_{k}\right)=\mathbf{X}_{a}^{a b s}+$ $\left[\mathbf{T}_{\text {vol }}\left(\mathbf{X}_{a}^{a b s}\right)\right] \delta \mathbf{X}_{a, \text { surf }}$. Again, the mesh deformation operator $\mathbf{T}_{\text {vol }}\left(\mathbf{X}_{a}^{a b s}\right)$ only depends on the absolute grid coordinates $\mathbf{X}_{a}^{a b s}$. This process is repeated at each iteration of the optimization process given an updated set of design variables. This process is illustrated in Figure II-11 below.

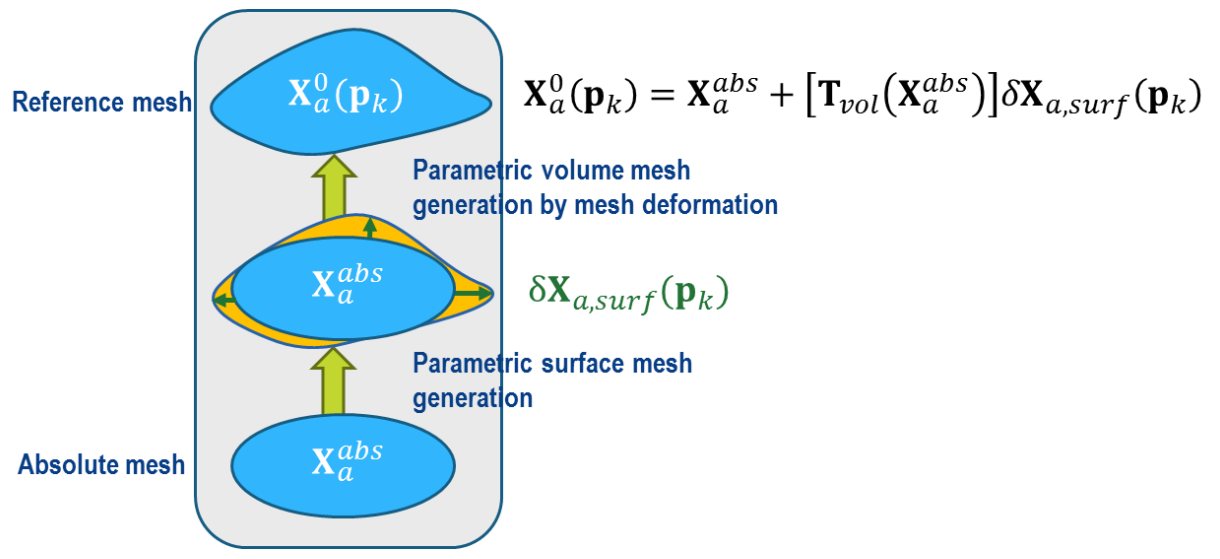

Figure II-11: Generic process for the generation of a parametric CFD grid. 


\section{Applications}

\section{ARTEMIS project (2009-2012) - Bi-level aero-structural optimization}

The optimization of aircraft configurations using standard disciplinary optimization algorithms and highfidelity numerical tools is today mature enough to be considered for the real aircraft design process by aircraft manufacturers. Among the challenges that arise today is the capability to design optimal multidisciplinary feasible configurations within a single global design process run. In this section a bilevel and bi-disciplinary optimization, based on a BLISS-like algorithm, of the Airbus XRF-1 transonic wing-body configuration is presented. The aero-structural optimization process shows that it is possible to improve the performance of the design while maintaining the feasibility for both aerodynamic and structure disciplines at each step of the optimization algorithm.

In the specific case of conventional transonic aircraft design aerodynamic and structural performance are two critical aspects. The strong interactions between these disciplines have motivated the development of high fidelity models, based on CFD for Aerodynamics and CSM for structures, as well as coupling capabilities by considering flexible wing computations. This early work intended to demonstrate the possibility to perform an aero-structural optimization of a wing-body transonic aircraft configuration using high-fidelity models for performance and sensitivity assessment integrated in a bi-level nonsequential process. Similar work on aerostructural MDO can be found in literature but are often based on different algorithmic approaches.

The work presented here was part of a more general framework named ARTEMIS (Advanced R\&T Enablers for Multi-disciplinary Integrated Systems) in collaboration with the Airbus company [14][15]. The MDO approach which has been chosen is based on the original Bi-Level Integrated System Synthesis (BLISS) system decomposition algorithm formulation proposed by Sobieszczanski-Sobieski [16]. This algorithm decomposes the problem into a global system optimizing the aircraft in a design space shared

by both disciplines and sub-systems, each of which will find an optimum with regard to a single discipline in its own dedicated design space. The consistency of the whole process relies on coupled-adjoint sensitivity analyses to formulate the separable disciplinary optimization problems and also on the postoptimal analysis tool to link the system and disciplinary sub-problems. Among the various advantages of this method, it allows each disciplinary framework to remain autonomous and to evolve without consequence on the other disciplines as long as the interdisciplinary consistency aspects are maintained.

An overview of the global process is presented in Figure II-12. The higher level is dedicated to the Overall Aircraft Design (OAD) and is responsible for the optimization of the global aircraft performance for a complete mission profile. The typical models used at this level are considered as low fidelity models and consist in polar curves, mass breakdowns, statistical data, response surface models. The intermediate level focuses on the aero-structural optimization of the aircraft configuration and performs high-fidelity fluid and structural analyses and gradient computations. The test case selected for this study is the Airbus XRF-1 glider configuration. The structured CFD mesh designed for RANS computations is made of 1.7 M cells over 143 blocks.

At the upper OAD level, the wing planform shape is designed (typically sweep angle, span, outer wing taper ratio, relative thicknesses). At the disciplinary level, the aerodynamic task is responsible for airfoils and twist law optimal design (several hundreds of design variables, geometry and global performance constraints) while the structural task optimizes the wingbox stiffness by varying composite skins and spars physical properties (several hundreds of design variables, up to 20000 design constraints). 

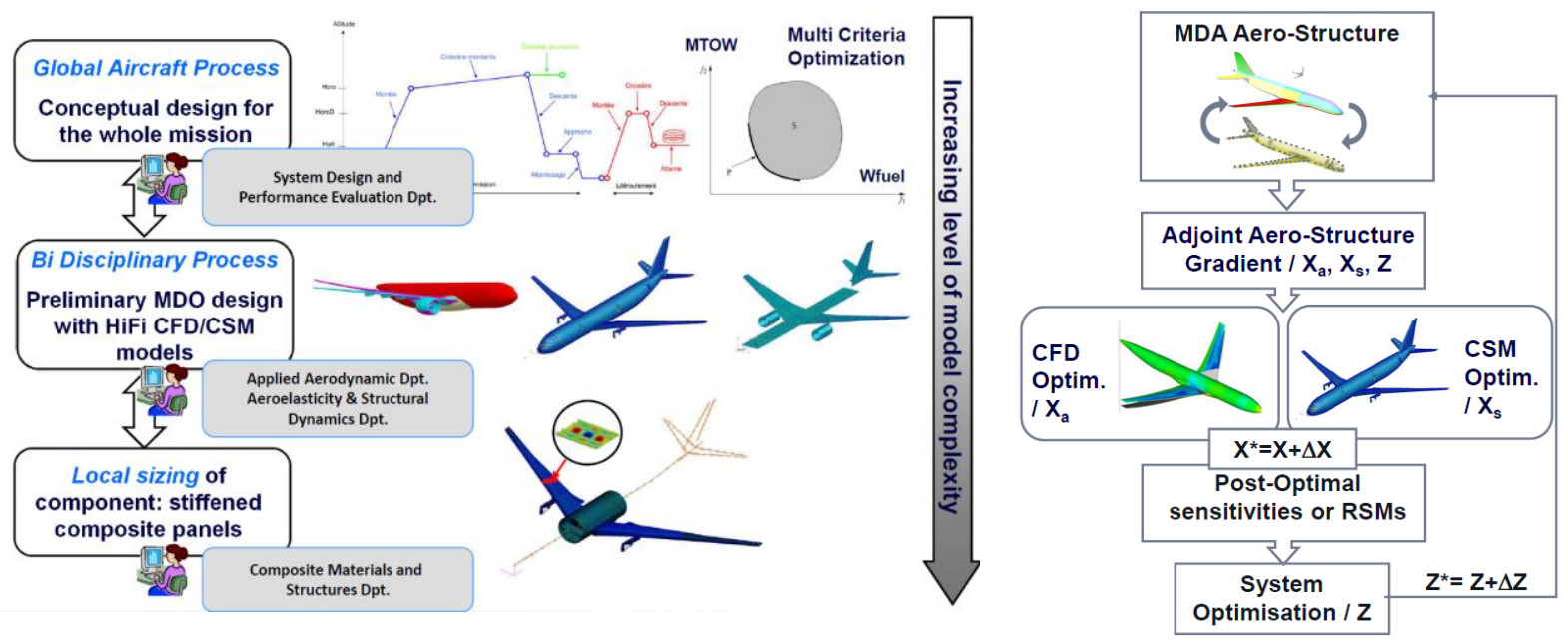

Figure II-12: Organization of the multi-level optimization process (left), bi-disciplinary process at the medium-fidelity level (right).

Additionally, the structure disciplinary design task embeds a critical loads computation module which is responsible for providing the correct set of aeroelastic sizing loads (see Figure II-13). The wing box design is performed using a bi-level scheme. At the upper level, only macroscopic parameters and constraints are addressed while the lower level is dedicated to the assessment of buckling criteria. The buckling reserve factors are then approximated using a response surface approach which is then fed back to the macroscopic wing design level. The complete flexible wing optimization workflow sequence is schemed in Figure II-14 (left part). The high-fidelity CFD computations are performed using the elsA software. The first step of the process is the mesh generation, performed using an in-house analytical mesh deformation tool, SeAnDef. The engineering parameters modifications are translated into skin deformation of the jig shape of the baseline configuration and propagated in the volume of the structured multi-block mesh. SeAnDef also provides the sensitivities of the mesh to the design variables, which are needed to assemble the final gradients.

A beam-like reduced-order model for the wing structure of the baseline is provided by the structure discipline and its characteristics are kept constant during the flexible wing aerodynamic optimization. Once the new mesh is made available an aeroelastic computation is performed using the elsA/AEL capability (aeroelastic module of $e l s A$ ) providing the equilibrium flow solution. The resulting flight shape is illustrated in Figure II-14 (top right). The near field flow analysis provides the aerodynamic performance coefficients needed by the optimizer, i.e. the lift coefficient $C_{L}$ and the pressure drag coefficient $C_{D p}$. The flow solution is also post-processed with the ONERA/FFD72 [17] far field drag extraction software which provides the phenomenological total drag decomposition into the wave, friction, induced and viscous pressure drag components (see example in bottom right of Figure II-14). Even if the optimization only deals with pressure drag reduction, the remaining components behavior is monitored through the process. FFD72 also calculates the sensitivities of the objective function and constraints with respect to the flow and the mesh which are also needed for the gradient assembly. 


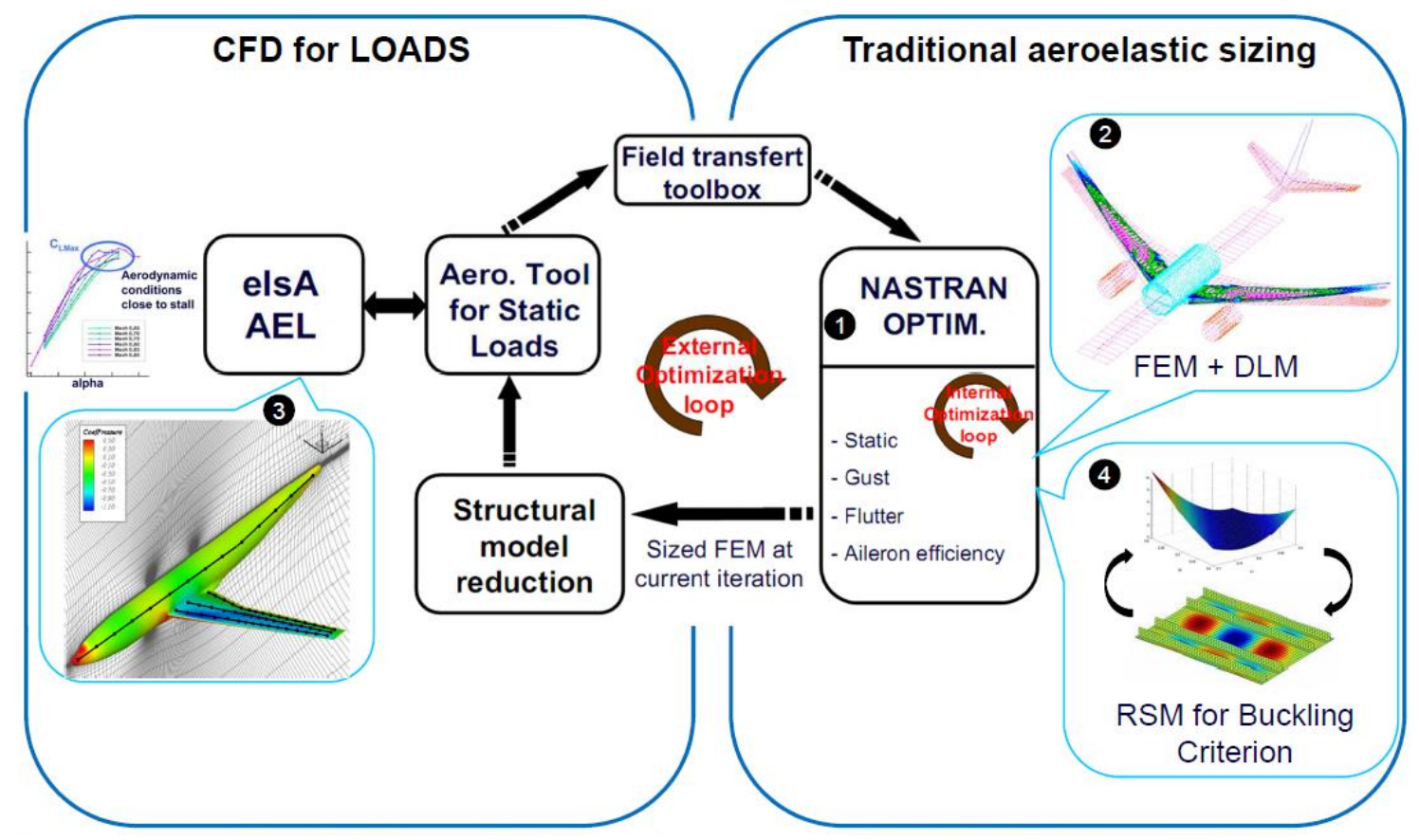

Figure II-13: Structural sizing workflow.
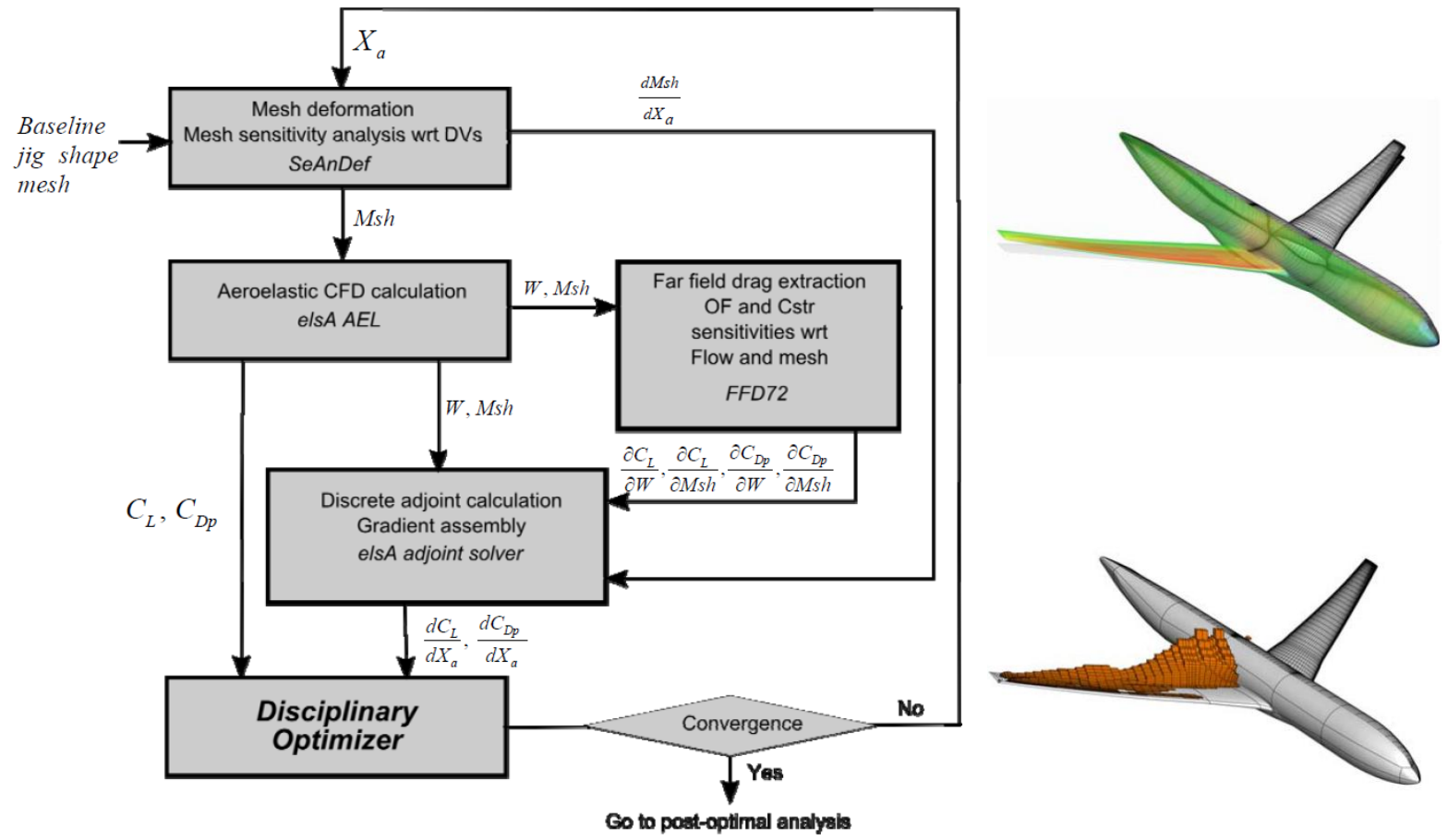

Figure II-14: Aerodynamic optimization workflow.

At the outcome of the disciplinary optimization processes, the set of Lagrange multipliers associated to active design constraints is computed and this post-optimal information is used to build the system level 
optimization problem. Then, once the bi-level problem has converged, the OAD low level models are updated according to the high fidelity data and a new aircraft configuration is designed.

A typical convergence for the aerodynamic and structure merit functions is presented in Figure II- 15 . Vertical lines indicate system-level optimization steps where the wing planform is designed. The inclined lines correspond to the variation of the sub-problem objective functions during the aerodynamic and structure optimization processes.
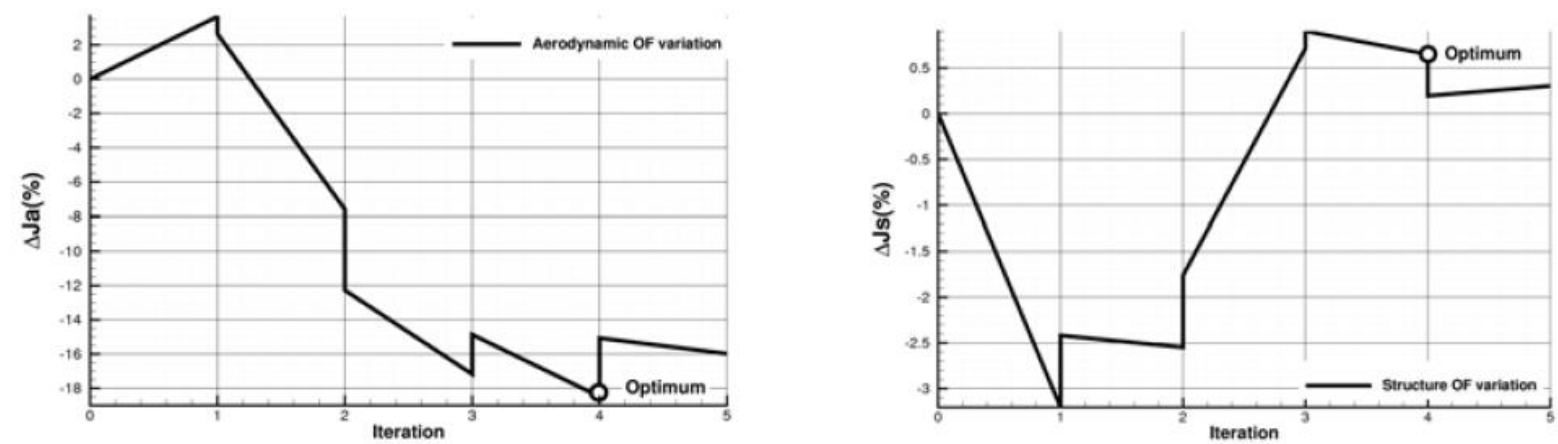

Figure II-15: Sub-system level objective functions history: structural wing weight (left) - pressure drag coefficient (right).

The figure below presents the overall merit function history at the system level. The evolution in terms of pressure drag contribution only and total drag contribution are compared. The optimal compromise is achieved in several iterations. The OAD level then updates the global aircraft arrangement by considering the performances along the entire mission profile and the overall process is repeated.

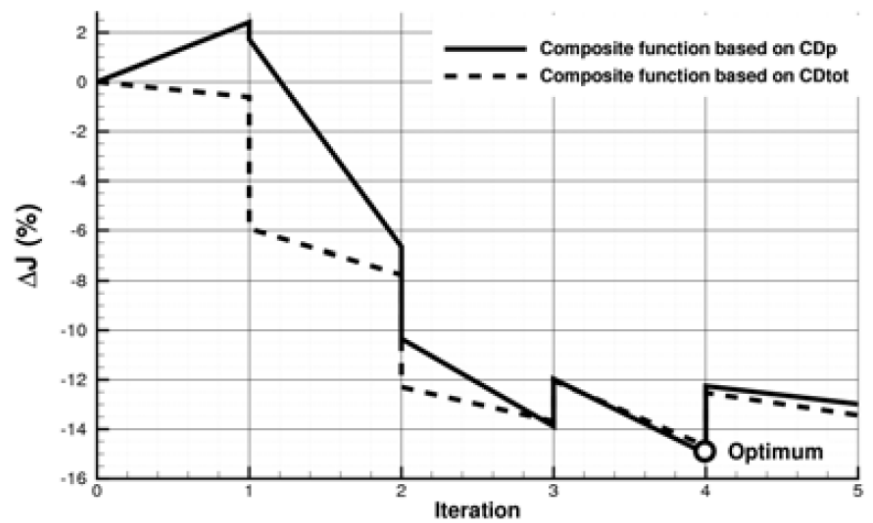

Figure II-16: System level objective function J (plain line) and total drag based composite function (dotted line) history through the optimization process.

\section{EU project CRESCENDO (2009-2012) - Coupling by design space}

A realistic application of advanced structural and multi-objective optimization for the design of a fully assembled aircraft powerplant installation is presented. As opposed to the classical design process of powerplant installation that does not consider the influence of pylon sizing over engine efficiency, we develop in the present work a fully integrated approach where both pylon and compressor intercase are designed at once. The main objective is to consider the impact of weight over tip clearance performance criterion and see how these two objectives are antagonistic. In this work, we perform in the same design 
session tasks traditionally devoted to the airframe manufacturer and aeroengine manufacturer. The overall weight of the assembly is minimized with respect to Specific Fuel Consumption (SFC) criterion. One major phenomenon to consider in both pylon and engine design is Fan Blade Off (FBO) event, i.e. the sudden release of a blade. This event causes high impact loads and must be considered carefully in the design. Such a simulation is not an easy task and several nonlinear phenomena must be addressed (e.g. rotor dynamics), not to mention the integration of this nonlinear dynamic response in a static structural optimization process. The interested reader can refer to [18] where a comprehensive description of this design study is proposed.

This multi-disciplinary study considers structural, aerodynamic, thermo-mechanical and fan-blade off event high-fidelity simulations. The pylon component is subject to critical loads for sizing the structural box while the design of the shape of the pylon fairing uses aerodynamic analyses for drag reduction. In this type of multi-physic design, there is no strong coupling through the disciplinary states. The coupling occurs mainly through the design variables that affect the behavior of each discipline. In this study the coupling between the engine case design and the pylon box design was considered through the stiffness of the engine intercase. From the specification of the design space, a response surface model of the SFC was built and introduced in the structural sizing process including the pylon critical loads from the FBO event, gust and steady maneuver load cases.

Figure II-17 below shows the powerplant assembly including the engine case, the pylon and the nacelle. On the right-hand side the design parameters controlling the stiffness of the engine intercase are identified by colored areas. The response surface model of the SFC criterion was built from a design of experiments with respect to these 13 design parameters. Each point corresponds to a full thermo-mechanical simulation of the engine. The SFC versus weight Pareto front is depicted in Figure II-18. A dedicated weighting approach has been used to draw points evenly distributed on the Pareto front.
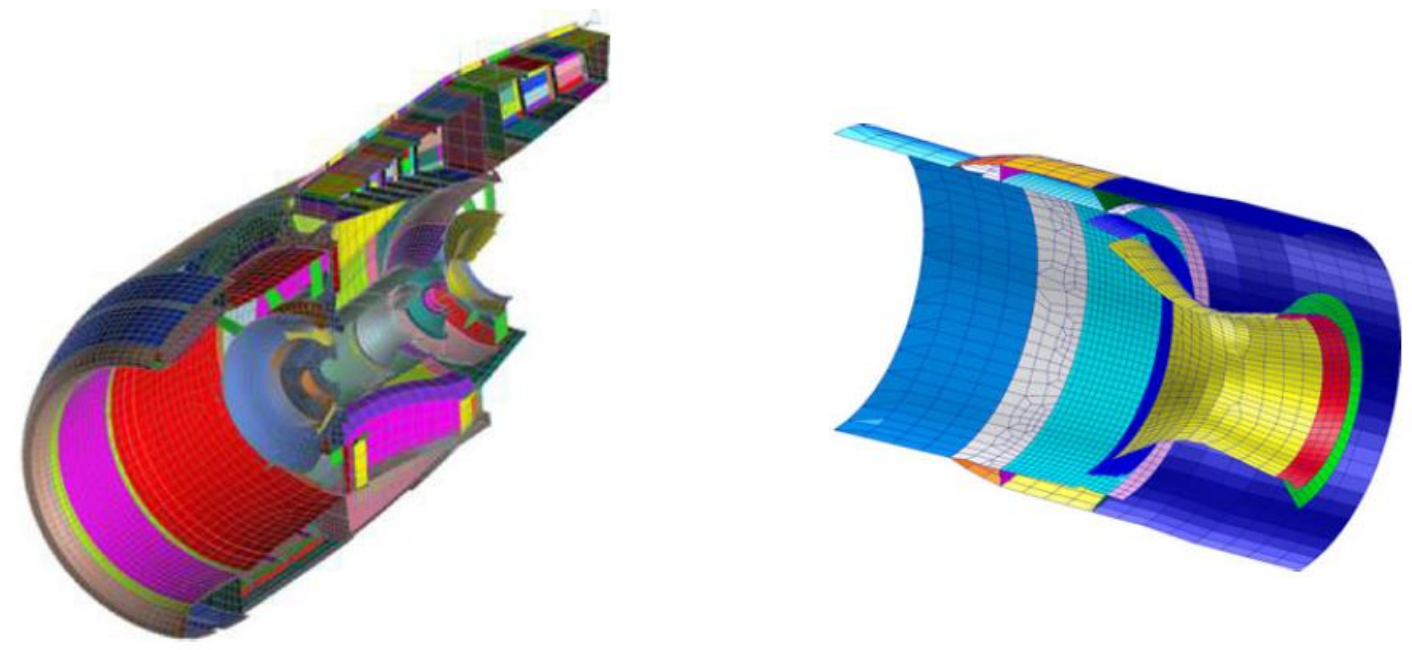

Figure II-17: Powerplant assembly (left) and engine intercase (right, colored by design areas).

We observe that we almost span uniformly the utopia line, that is the line between the two individual optimums, which would indicate that we do not miss large parts of the real Pareto front. From numerical tests (signature of approximated Hessian matrices for uniformly distributed points in the design space), both objective functions appear to be convex over design variables. The fact that some scalar optimizations did not converge to a Pareto-optimal solution does not necessarily infer the convexity of objective functions. 


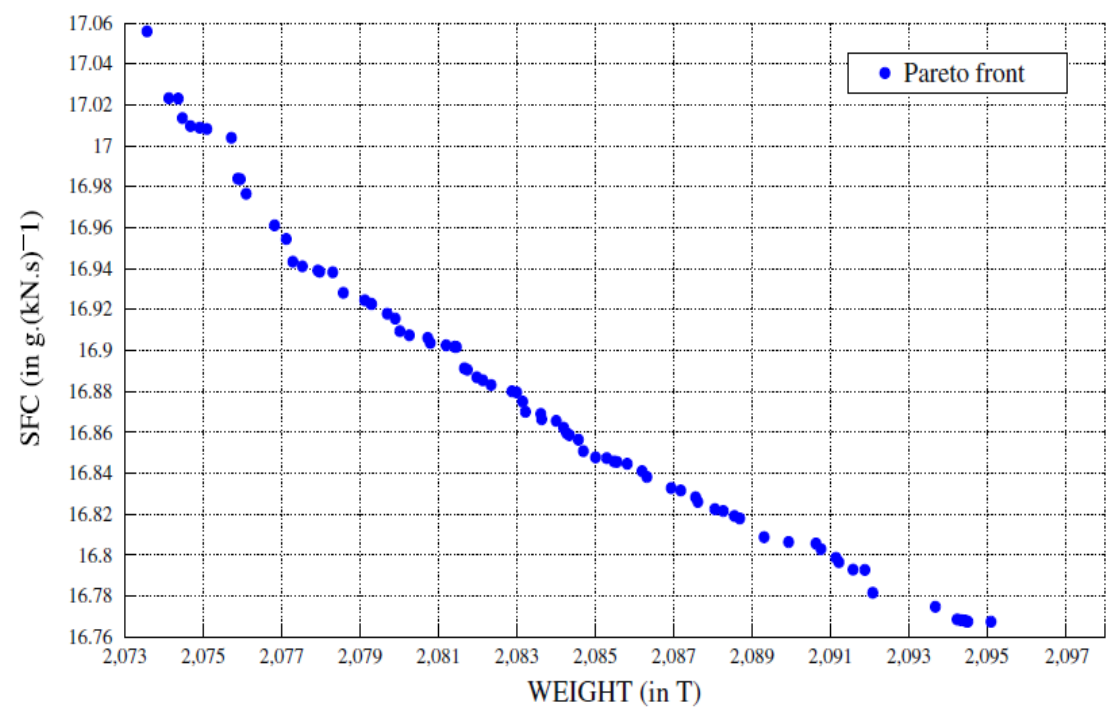

Figure II-18: SFC vs. pylon weight Pareto front.

Indeed, some convergence issues and numerical inaccuracies can also explain why for some values of the weighting coefficient $\alpha$, the objectives at convergence are not Pareto optimal but they are, however, quite close to the front. We observe that a decrease in weight of $22 \mathrm{~kg}$ increases SFC by about $0.3 \mathrm{~g} \cdot(\mathrm{kN} \cdot \mathrm{s})-1$. This amount of SFC represents for instance for a standard engine for Airbus A320 of $120 \mathrm{kN}$ of thrust for a one-hour flight more than 150 liters of fuel. From this final Pareto front, we observe that engine (or at least compressor intercase) flexibility has a quite negative impact on overall aircraft performance. This indicates that the flexibility of the engine must be considered to efficiently predict and validate SFC. From the airframe manufacturers point of view, such a front of potential pylon-engine designs is useful to select a pylon design by evaluating its impact over engine performance.

3. EU project MADELEINE (2018-2021) - Aero-structural wing optimization applied to a large passenger aircraft configuration

The concept proposed in MADELEINE [10][11] aims at demonstrating the benefits of using high-fidelity, robust adjoint-based optimization processes to address multi-physics problems, with the final objective of industrial usage by designers in the aeronautic industry. The main results of the project will provide to designers, a new way of working between disciplinary experts and a new insight for design space exploration and the definition of better multi-disciplinary compromises.

The main objective here is to apply and demonstrate the maturity of the multi-disciplinary aero-structural adjoint formulations, developed during the project, on complex relevant industrial test cases and conclude regarding the main bottlenecks in order to guide future developments. More specifically, the industrial test case of interest here has been defined by Airbus Operation SAS around the XRF-1 model, a representative large passenger aircraft. In this task, Airbus sets-up real industrial test cases in terms of geometry, operational points and constraints, in order to be able to draw reliable conclusions that can guide the industry for future applications of MDO using the adjoint approach. The major partners involved in this work are Airbus, DLR and ONERA. 


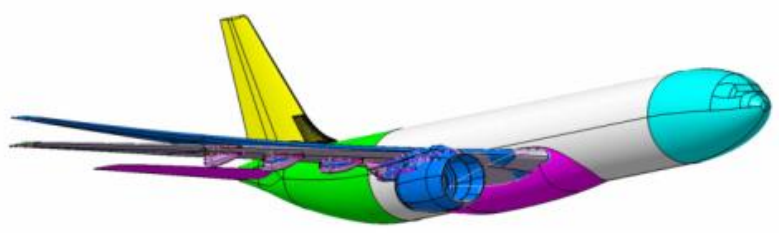

Figure II-19: XRF-1 full aircraft configuration

Before performing complex aeroelastic and aero-structural optimizations, all partners agreed to first validate the implementation of multi-disciplinary adjoint capabilities on a simplified XRF-1 wing-body configuration. In order to ease the validation of sensitivities between partners, some data and disciplinary bricks have been shared between partners whenever possible. All partners used for instance the same CSM finite element model (provided by DLR), and put effort in using the same wing shape parametrization with 13 parameters, selected to be representative of a wing optimization exercise: span, sweep, chord lengths, camber, thickness and twist of airfoils. This number of parameters is for sure too low for wing optimization, but was used for demonstration and validation purpose only. The multidisciplinary optimizations will be performed on much higher number of design parameters to demonstrate the benefit of the adjoint approach.

At first, partners compared rigid and flexible gradients computed with their own in-house tools. Also to make the comparison relevant, all sensitivities were first validated internally by comparison with central finite differences.

The flow conditions retained in this study correspond to standard cruise flight conditions. For the structured grid we use the elsA CFD software. A viscous RANS aeroelastic analysis is performed with a Jameson centered spatial discretization scheme and a Spalart-Allmaras one equation turbulence model. The maximum wing tip displacement at the outcome of the aeroelastic analysis is equal to $1.279 \mathrm{~m}$ for a prescribed lift coefficient $\mathrm{C}_{\mathrm{L}}=0.50$, including the inertia relief contribution. The elastic twist increment distribution as seen by the structural wingbox centerline reaches up to -3.5 degrees of washout. The datum jig and flight aerodynamic shapes (provided by Airbus) along with the computed aeroelastic equilibrium configuration (in blue) are presented in Figure II-20. The current finite element model appears to be a little bit stiffer than the datum finite element model of the XRF1. However, achieving the same flight position as the reference model was not an objective for the current task. 

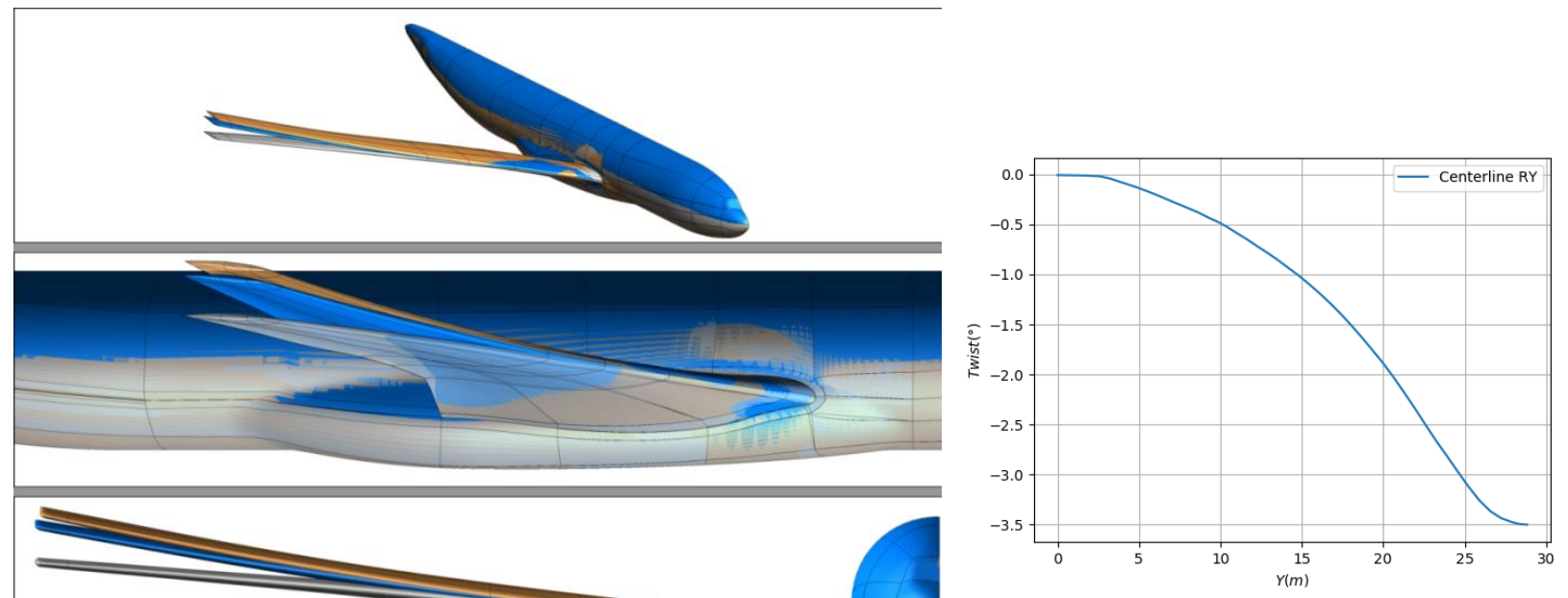

Figure II-20: Flight shapes computed by the aeroelastic analysis and associated elastic twist increment.

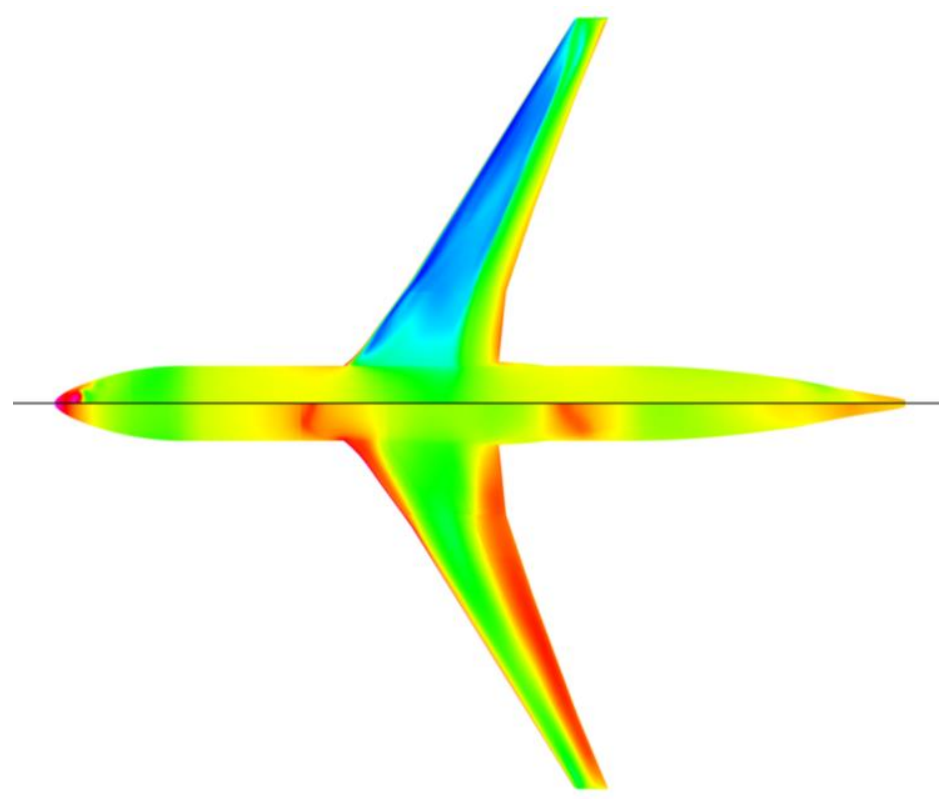

Figure II-21: Pressure Coefficient distribution (top $=$ upper surface, bottom $=$ lower surface) on the aeroelastic flight shape.

The definition of the 13 retained design variables is as follows: three airfoil sections are considered and are controlled by 3 local design parameters whereas global wing planform design parameters like span, sweep, crank and tip chord are also defined. Figure II-22 depicts the wing parametrization. The PADGE parametric CAD model contains 9 sections to fit the aerodynamic wall grid. The link between the 9 matching airfoil sections and the design variables is illustrated on the right-hand side of Figure II-22. The root section S0 is frozen. Section 3, 5 and 8 are controlled by the twist, thickness and camber design 
variables. In addition, section 3 at crank and section 8 at wing tip are allowed to change their chord length. Intermediate sections 1, 2, 4, 6 and 7 are then appropriately interpolated from the parametric sections.

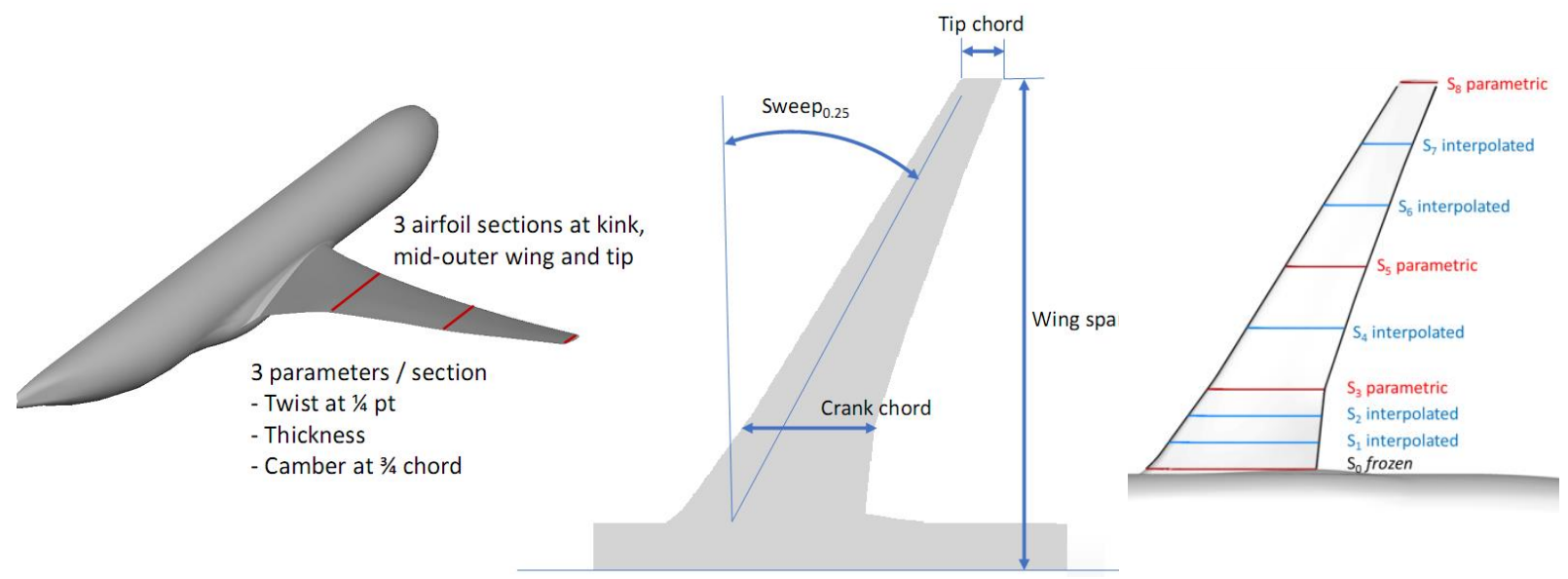

Figure II-22: XRF1 wing shape parameterization for aeroelastic sensitivity analysis.

The coupled-adjoint sensitivity analysis is then performed by solving the linearized equations around the computed flight shape. It is worth mentioning that a fully linearized turbulence model was considered for gradient computation.

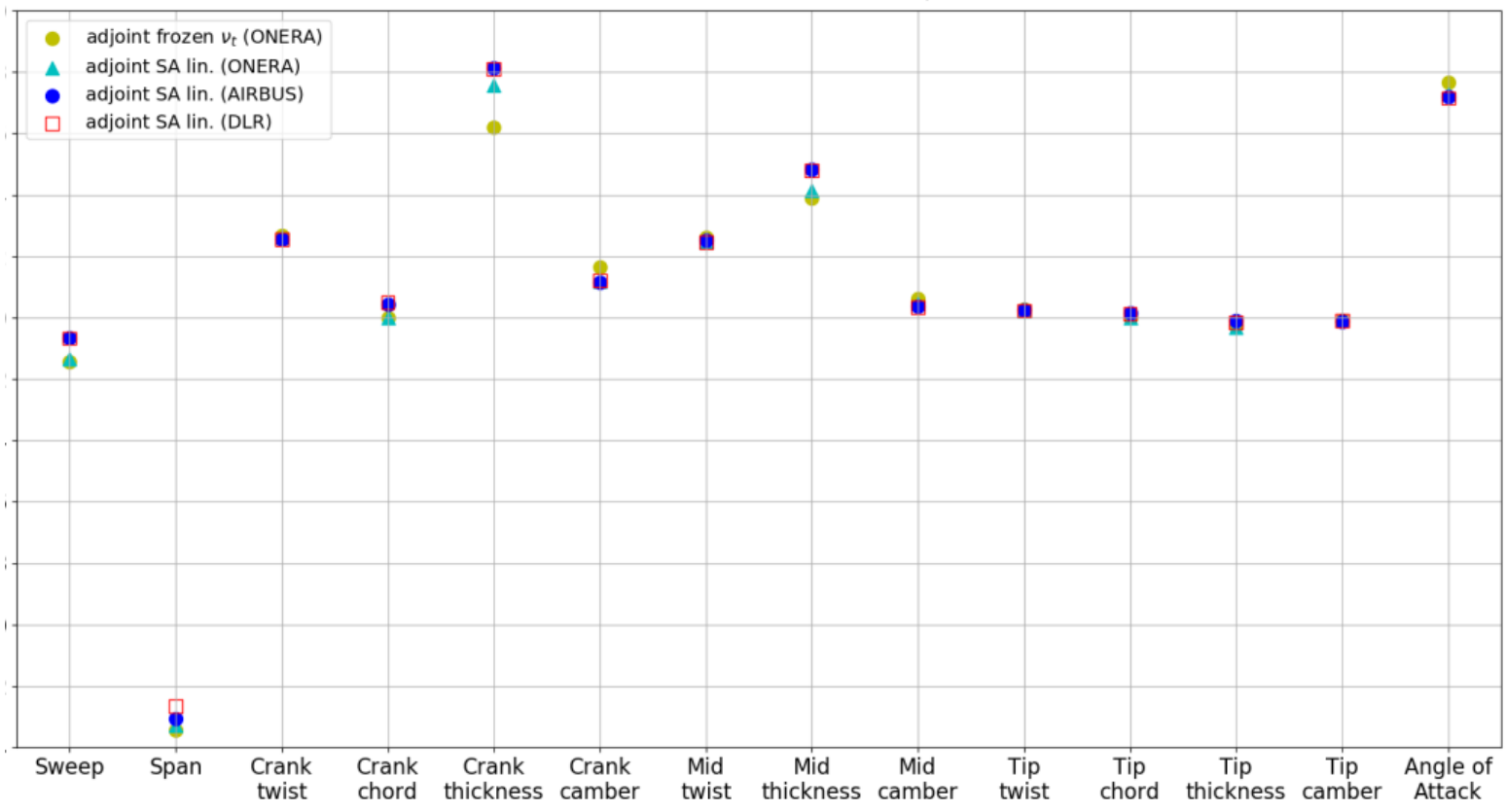

Figure II-23: Comparison between ONERA, DLR and AIRBUS of coupled sensitivities for Pressure Drag (CDp).

As a representative result, Figure II-23 presents a comparison between Airbus, DLR and ONERA coupled sensitivities for pressure drag computed in transonic flow. The agreement is particularly good, considering variability in mesh strategy, mesh deformation, CFD settings, etc. 
A more detailed comparison is also presented in Figure II-24 where rigid and flexible sensitivities of $\mathrm{CD}_{\mathrm{p}}$ are reported for the frozen eddy viscosity assumption and for the fully linearized Spalart-Allmaras turbulence model. First it can be observed that the turbulence model linearization can significantly affect the rigid gradient values, especially for the span and angle of attack design variables. However looking at flexible counterparts, this seems to be mitigated by the impact of structural flexibility, which is particularly important for planform design variables. From this cross-validation exercise partners were sufficiently confident in the maturity and accuracy of their coupled adjoint solvers which was an important milestone before addressing realistic optimizations.

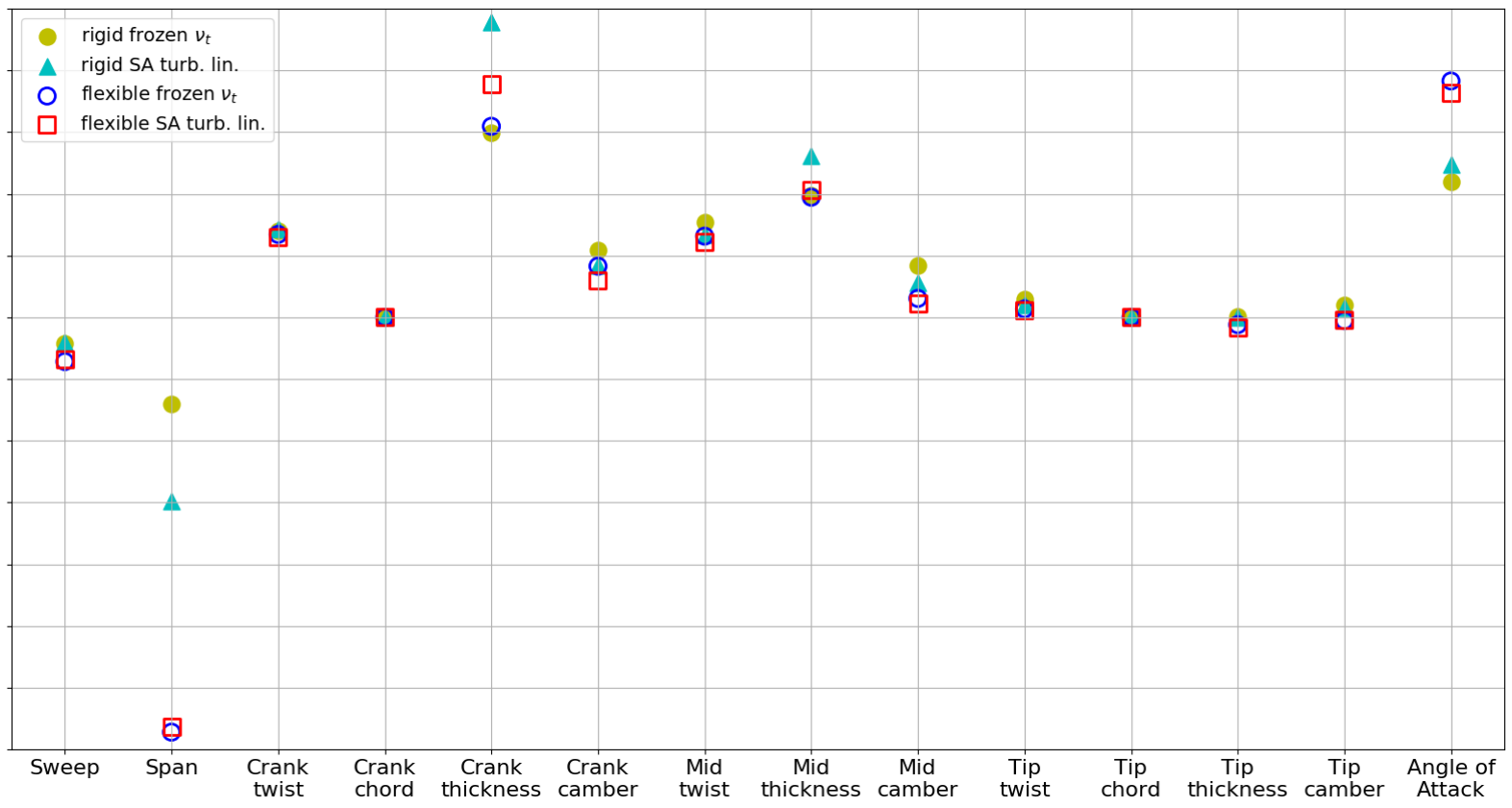

Figure II-24: Rigid vs. flexible derivatives for the pressure drag coefficient for frozen eddy viscosity assumption and for fully linearized turbulence model.

This work represents an important European collaboration between research institutes and the industry in the aim of leveraging new capabilities for preliminary aircraft design. Despite the variability in the numerical approaches used by the different partners we still achieved very fair comparison for coupled gradient values. More specifically we have demonstrated the added benefit in terms of accuracy of considering flexibility in the computation of sensitivities. In addition, it is now acknowledged by all partners that the fully linearized turbulence model should always be considered in gradient computations. In the final paper results about multi-point flexible optimizations and fully coupled aero-structural optimizations will be presented. 


\section{III.Example of aero-acoustics simulations for design}

An aero-acoustic simulation framework combining the ONERA elsA CFD solver and sAbrinA-v0 CAA solver has been developed and applied for the aero-acoustic setting optimization of the slat of a simplified (2D) three-bodies high-lift configuration at take-off conditions. These aero-acoustic simulations illustrate a "chained" simulation capability requiring independent, though consistent, mesh generation for the two disciplines, specific post-processing and interpolation of the CFD results to feed the CAA simulation (the mean aerodynamic field interpolations from the CFD mesh to the CAA).

The elsA CFD solver was used to perform 2D RANS simulations of a spanwise cut of a wing equipped with a deployed three-bodies high lift system. From this RANS simulation, the modeled turbulent kinetic energy in the slat cove region was post-processed to derive an equivalent noise source. The CAA sAbrin$v 0$, was then used to solve the non-linearized Euler equations (applied to the disturbances) in time domain, around the full geometry, taking into account non-uniform aerodynamic mean-flow from the RANS simulation and evaluating the noise radiated by the equivalent noise source.

\section{A. Parametrization}

The investigated parameters retained for the optimization correspond to a solid-body displacement of the leading-edge slat. The following three parameters are usually considered by designers to describe this displacement of the slat: its deflection angle, its gap and its overlap with respect to the main wing. However, it has to be noted that all of the \{Gap,Overlap\} combinations are not feasible with this parameterization: for instance when overlap is negative, points with gap value lower than (-overlap) are not geometrically possible (Figure III-1).

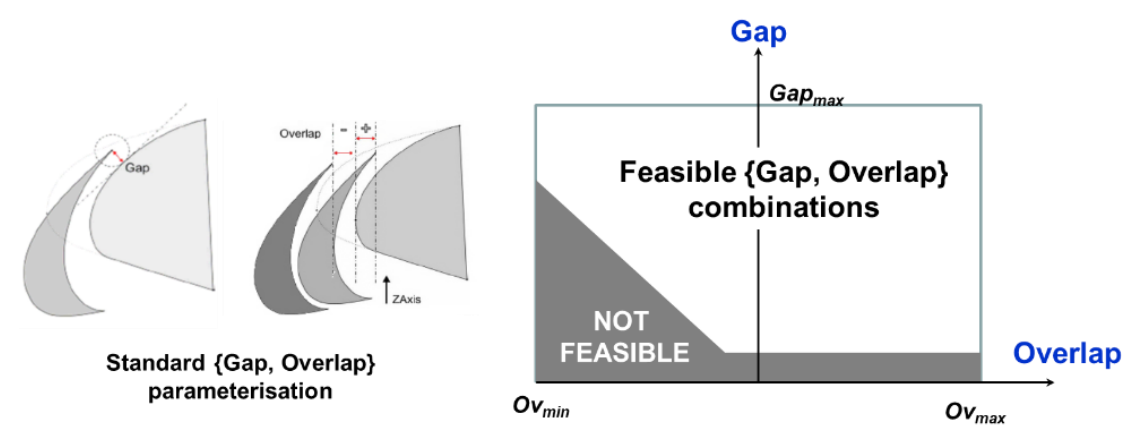

Figure III-1: Standard \{Gap,Overlap\} parameterization.

This is not a problem for the optimization problems based on direct evaluation of the cost functions, where this can be treated by the use of threshold values (see [21] for instance). But when considering meta-models, it is highly recommended to have the information within all the design space for the accuracy of the models. Several methods are possible to overcome this problem one of them is to use the polar coordinates $(\rho, \theta)$ instead of the (Gap, Overlap). The advantage of such parameterization is that all the combinations are possible in the $(\rho, \theta)$ space, which is of high importance for the accuracy of surrogate models (Figure III-2). 


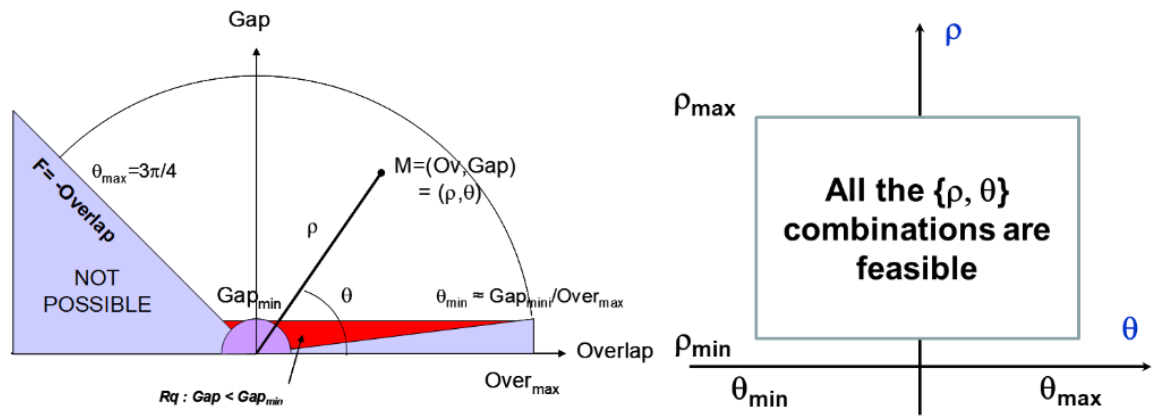

Figure III-2: Polar $\{\rho, \theta\}$ parameterization.

Relationship between settings variables is simply:

- $\operatorname{Gap}=\rho * \sin \theta$

- Overlap $=\rho^{*} \cos \theta($ with $\theta>0)$.

In this parameterization, the limit for feasible solutions, which is the line Gap=-Overlap, corresponds to $\theta_{\max }=3 \pi / 4$. For practical reasons (mainly grid generation), we can fix some Gap $\min$ and an Overlap $\max _{\max }$ values. In the present optimization problem considered, the following extreme values have been used :

- Minimum gap: $1.5 \%$ and Maximum overlap: $+7.5 \%$. These values lead to:

$$
\begin{array}{ll}
\circ & \rho_{\min }=\text { Gap }_{\min }=0.015 \\
\circ & \rho_{\max }=\text { Overlap }_{\max }=0.075 \\
\circ & \theta_{\min }=\frac{\text { Gap }_{\min }}{\text { Overlap }_{\max }}=0.20 \\
\circ & \theta_{\max }=\frac{3 \pi}{4}=2.356195
\end{array}
$$

- For the deflection angle, variations with respect to the initial setting have been considered: $\delta=\delta_{0}+\Delta \delta$, with $\Delta \delta \in\left[-2.5^{\circ},+12.5^{\circ}\right]$

Finally, a scaling procedure is applied in order to have design variables $\left(\mathrm{x}_{\rho}, \mathrm{x}_{\theta}\right.$ and $\mathrm{x}_{\delta}$, respectively linked to $\rho, \theta$ and $\Delta \delta$ ) set between 0 . and 1 .

\section{B. Optimization problem}

The optimization problem considered in this task consists in optimizing the slat settings of a twodimensional high-lift configuration according, simultaneously to different performance criteria: the aerodynamic lift (which is to be maximized at flight operating and maximum lift conditions, and the aeroacoustic noise radiated downward by the high-lift device (which is to be minimized at the flight operating condition). Since these criteria are a priori uncorrelated, this problem has to be considered as a generic multi-objective optimization problem in which one seeks to identify the Pareto front for the considered cost functions, i.e. the set of non-dominated designs with respect to the Pareto order relation. By non- 
dominated, we mean that there is no better design being strictly better simultaneously for both objectives. The mathematical formulation of the optimization problem is therefore as follow:

Find the vector $\vec{X}=\left[X_{\rho}, X_{\theta}, X_{\delta}\right]$, which is Pareto optimal with respect to:

- Obj_Aero_20 : Increase lift at $M=0.168, \operatorname{Re}_{C}=12.510^{6}, \alpha=20^{\circ}$;

- Obj_Aero_5 : Increase lift at $\mathrm{M}=0.168, \operatorname{Re}_{\mathrm{C}}=12.510^{6}, \alpha=5^{\circ}$;

- Obj_Acou_5 : Minimum noise at $\mathrm{M}=0.168, \operatorname{Re}_{C}=12.510^{6}, \alpha=5^{\circ}$.

It corresponds to a tri-objective optimization problem, with the following cost or objective functions:

- For the aerodynamic problems, the objective functions are the relative improvement of lift coefficient at the corresponding angle of attack, compared to the reference configuration:

$$
\text { obj_aero }=-100 \times \frac{C_{L E v a l}-C_{L R E F}}{C_{L R E F}} .
$$

As most of the optimizers use some minimization algorithms, the sign "minus" is used in the cost functions considered (an improvement of $2 \%$ of lift gives obj_aero=-2).

- For the acoustic problem, the objective function to minimize is defined as the noise level (in $\mathrm{dB}$ ) estimated from the acoustic analysis module, relative to the reference configuration:

$$
o b j_{-} \text {acou } 5=\frac{\text { Noise }(d B)}{\operatorname{Noise}(d B)_{R E F}} .
$$

Therefore, improvement of acoustic performance leads to obj_acou5 $<1$.

\section{Optimization methodology}

ONERA has consolidated over the past years several experiences in the field of high-fidelity multi-criteria design optimization. Considering this expertise and the characteristics of the design problem at hand (cost of the analyses, number of design parameters and expected behavior of the optimization functions), a specific optimization methodology using Surrogate-Based Optimization (SBO) has been selected to conduct the present study. This SBO approach is intended to reduce the number of exact and costly CFD and CAA simulations necessary during the optimization (compared to a direct optimization of the exact criteria). Note that another advantage of the use of SBO is that each models can be based on different sampling points (the only constraint is to use the same parameterization).

As described in Figure III-3, SBO proceeds by first generating mathematical surrogate models over the complete design space of each of the optimization criterion involved in the optimization problem. Based on the high-fidelity database, one model is built-up for each objective function considered. In order to improve the model quality, the model is updated by the "expected improvement" points. 

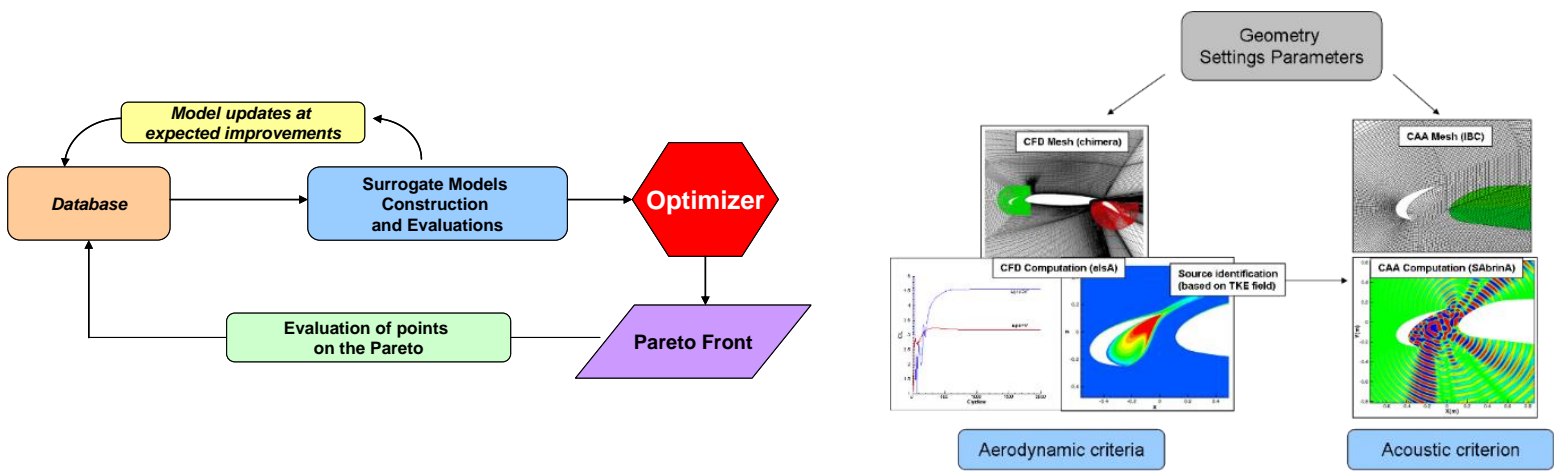

Figure III-3: Flowchart of the SBO method used

Then, an optimization of the approximated criteria are conducted using the surrogate models and, eventually, these surrogate models are enriched with the new designs issued from the solutions of these approximate optimization problem, once the exact values of their optimization criteria have been recomputed with the exact models and if these exact values confirm the optimality of the design. An iterative loop performing successively the optimization of the approximate criteria and the surrogate models enrichment is finally conducted until convergence.

\section{Optimization results}

Figure III-4 presents the different models obtained at the end of the updating process with surfaces of isovalues of objective functions in the design variable space. The final sampled points (yellow symbols) were necessary in order to improve the accuracy of the Aero_20 model in the optimal area. At the end of the model construction phase, 75 high-fidelity CFD evaluations have been considered. For the acoustic model, only 55 CAA evaluations were necessary. Based on these surfaces, it can be seen that the Aero_20 problem will probably restrict the field of solutions for the global optimization problem

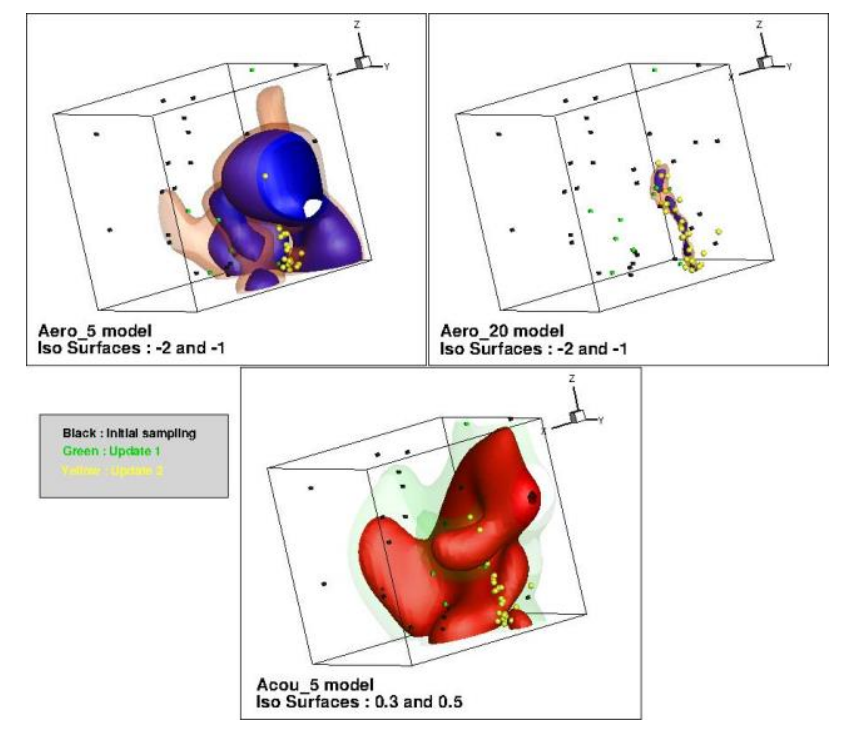

Figure III-4: Surrogate models used for optimization 
Figure III-5 presents the Pareto front of the tri-objective aero-acoustic optimization problem in the space of cost functions and of design variables. The red symbol corresponds to one of points used for the last update of the surrogate models using high fidelity methods. Though it is not strictly located in the Pareto front obtained, it can be considered representative of the solutions, and has been retained as the optimized configuration for the current problem.

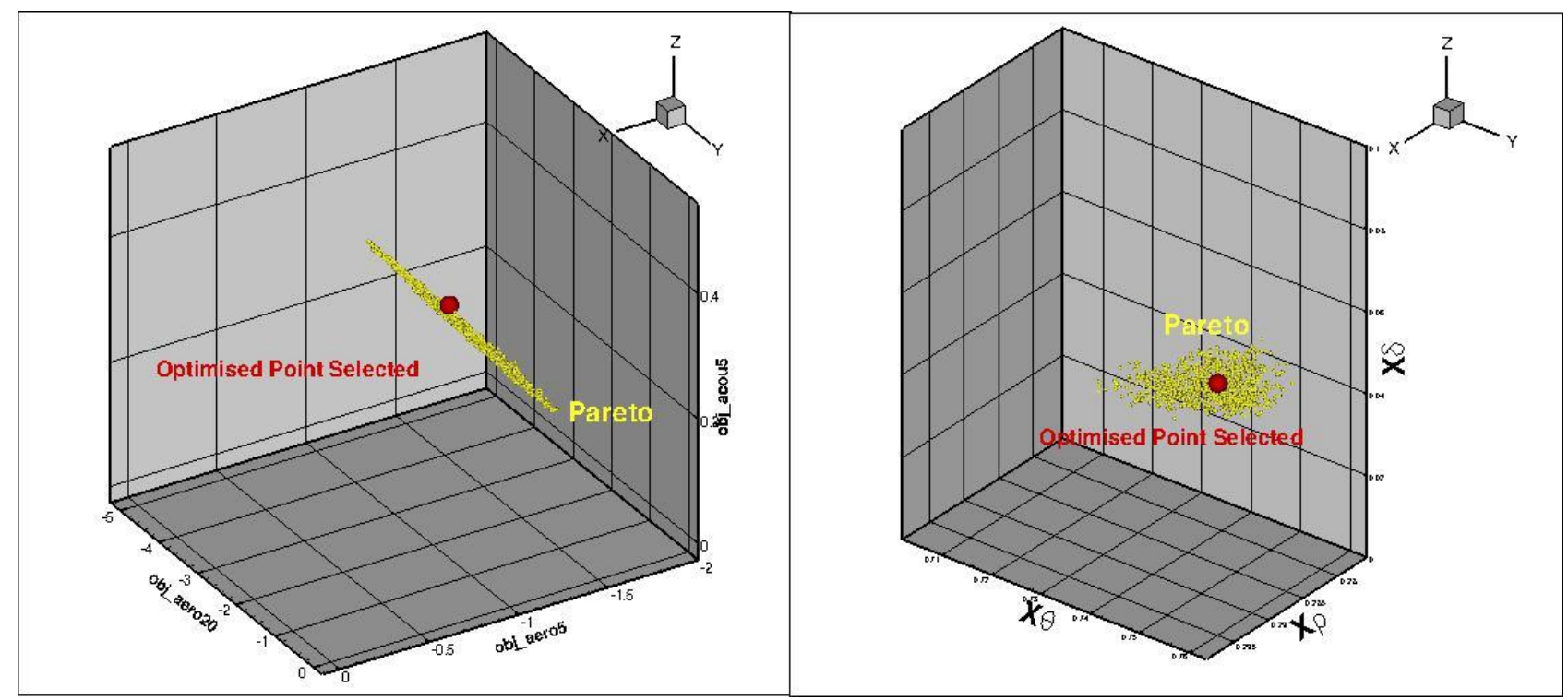

Figure III-5: Pareto front of the aero-acoustic problem and optimized point selected.

Table 1 compares the settings of the initial (reference) configuration with the optimized ones. The values of the different cost functions are given. In term of aerodynamics, the local performance has been improved by about $1.2 \%$ at flight conditions, and by $2.8 \%$ close to $\mathrm{C}_{\mathrm{Lmax}}$. In terms of acoustic source, the slat noise source has been reduced by about $70 \%$. Of course, for an extrapolation of the benefits to the complete aircraft, the balance of the difference acoustic sources to the total noise has to be considered.

Table 1 Comparison of the initial merit function values with the optimized ones.

\begin{tabular}{|l|c|c|c|}
\hline & Obj_Aero5 & Obj_Aero20 & Obj_Acou5 \\
\hline Initial & 0 & 0 & 1 \\
\hline Optimized & $-1.259 \%$ & $-2.789 \%$ & $0.296(-70.4 \%)$ \\
\hline
\end{tabular}

Figure III-6 compares the aerodynamic and the acoustic fields for the initial and the optimized configurations. A strong reduction of the maximum turbulent kinetic energy and acoustic levels is observed which leads to a significant decrease of noise emission in all directions, and more importantly in the downward direction. 

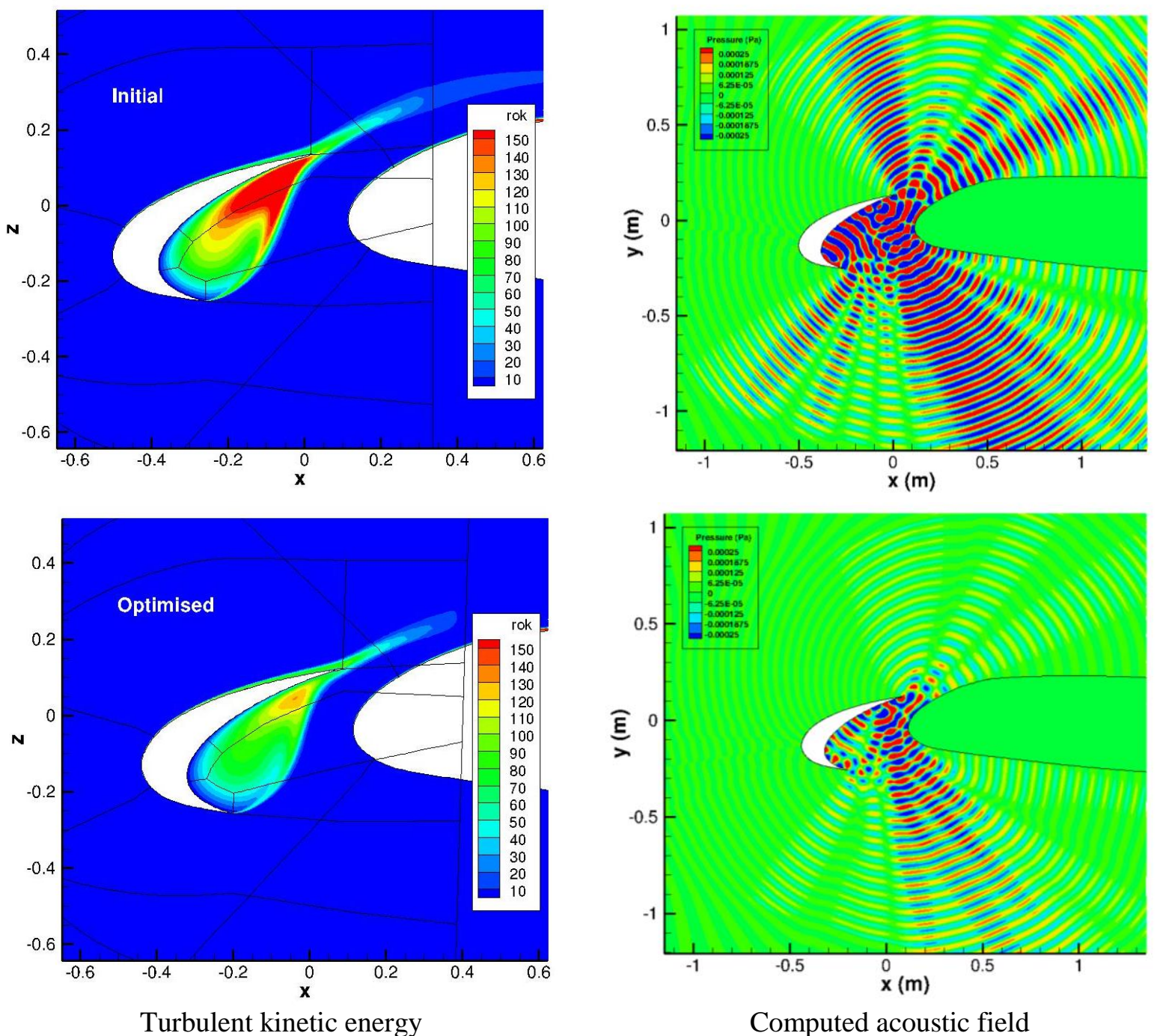

Figure III-6: Comparison of initial and optimized configurations - Turbulent kinetic energy and acoustic field at $\alpha=5^{\circ}$. 


\section{IV.Example of simultaneous aerodynamic and sonic Boom signature analysis and design for low-boom Supersonic Civil Transport}

\section{A. Description of the problem}

ONERA has initiated in 2020 a research effort combining conceptual design with a concomitant preliminary aerodynamic and low-boom design of a Supersonic Civil Transport Aircraft (SCTA). The aerodynamic efficiency in supersonic cruise conditions and the loudness of the sonic boom signature are commonly admitted to constitute antagonist objectives it the design of the aerodynamic shapes of an SCTA. Furthermore reducing the sonic boom by the aeroshaping of such an aircraft requires high degree of control on the aerodynamic surfaces, especially for the nose design. The current section presents the high-fidelity methods-based analysis system set-up in this study for simultaneously optimize the aerodynamic lift-to-drag ratio in cruise and the loudness of the sonic boom.

Based on existing market studies [22], recent and ongoing studies in industry and academia [23][24][25][26][27][28] (and a large a priori), the following Top Level Aircraft Requirements (TLAR) have been selected for this design studies:

- a range of $5000 \mathrm{Nm}$,

- a payload $16.000 \mathrm{lb}(\sim 80 \mathrm{pax})$,

- a low-boom cruise at a design Mach number of 1.6

\section{B. Methods and tools description}

One of the challenge of this design application is handling geometry. Indeed, it requires the capability to modify and parameterize the external aerodynamic shapes over the complete aircraft configuration with all its components including the propulsive system, with a high degree of freedom while taking into account top-level aircraft geometrical constraints for the cabin, cargo and landing gear bays. For that the Engineering Sketch Pad [29] geometry modeler has been used to generate a parametric geometric model of the complete aircraft and enable automated CFD mesh generation around the resulting aircraft shapes using the commercial mesh generator Pointwise and its automation capability based on Glyph scripts [30].

The evaluation of the aerodynamic performances in cruise condition relies on Euler CFD calculation using the SU2 CFD code [31] with added empirical evaluation for viscous drag. 


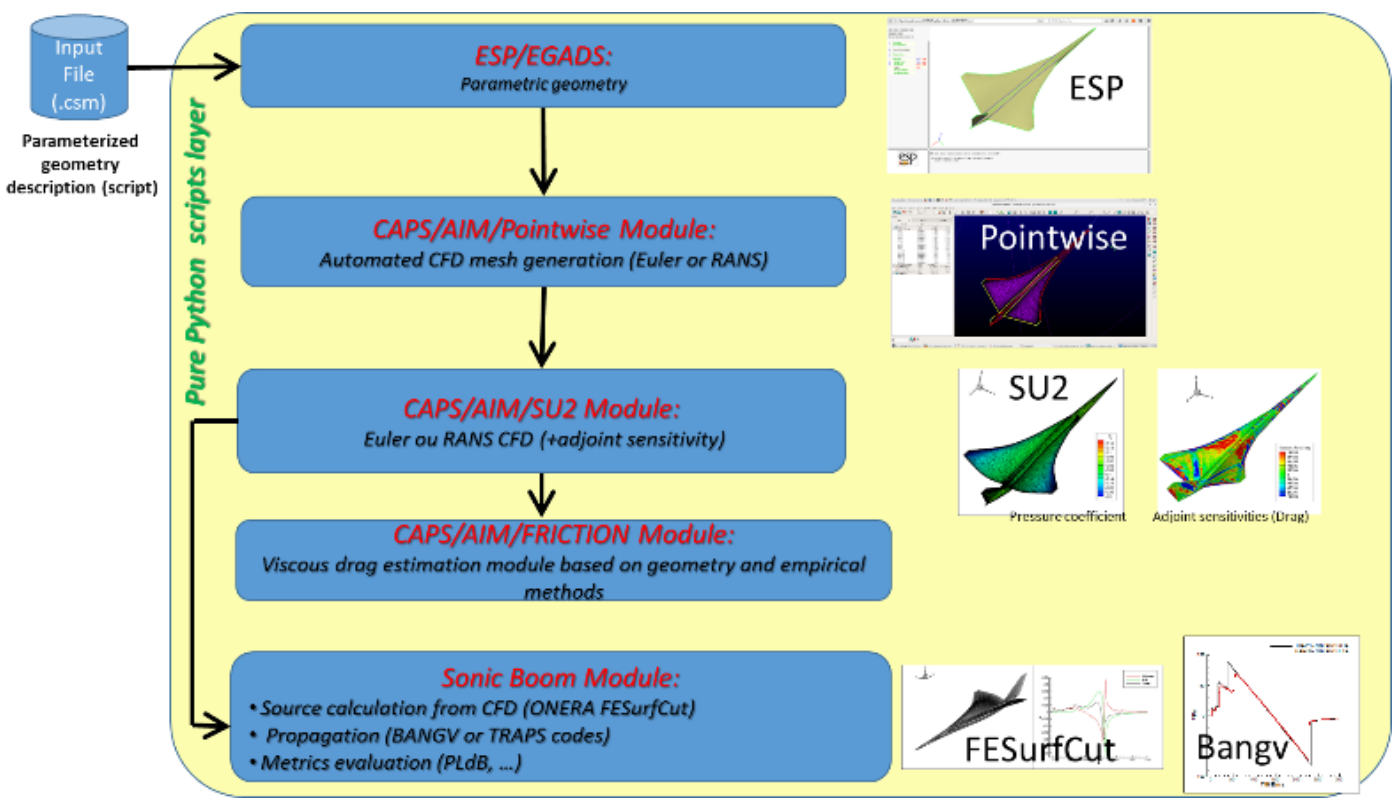

Figure IV-1: Description of the automated aerodynamic and sonic boom analysis system.

The complete MDA system developed for this aero-boom design application in depicted in Figure IV-1. Figure IV-2 illustrates one of its components, the sonic boom sources evaluation module. This module exploits the information of the surface CFD solution to calculate separately the volume and lift contributions to the so-called Whitham function that actually models the sonic boom sources in the nearfield. This Whitham function is then used as the input to a non-linear acoustic ray tracing code in order to propagate the sonic boom through a specified atmosphere and evaluate in the end the sonic boom at ground level and its loudness.

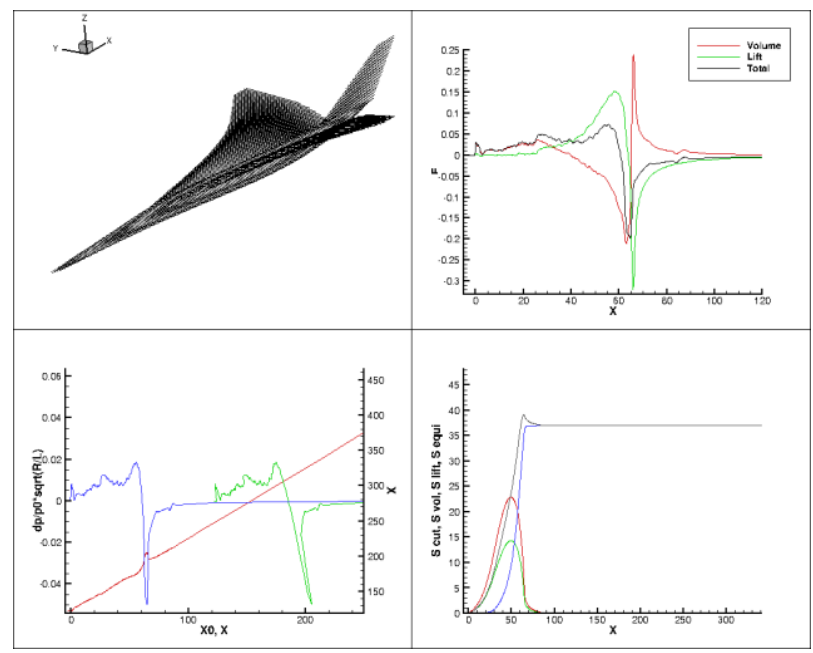

Figure IV-2: Evaluation of sonic boom sources from the near-field (surface) CFD solution using cross-sectional cuts in the geometry (top-left), equivalent area cross section calculation (bottomright) and derivation of the associated Whitham function (top-right). 


\section{Illustrations of analysis and design results}

Preliminary results have been achieved at the time this paper is written. They consist first in the verifications of the MDA system through two optimizations performed separately for the fuselage and wing (Figure IV-3). The first optimization used a direct optimization method (Nelder-Mead simplex) while the second used a gradient-based BFGS algorithm. These two optimization exercises demonstrated both the robustness of the complete MDA process with automated mesh generation and CFD calculation over a wide range of aircraft geometry variations.
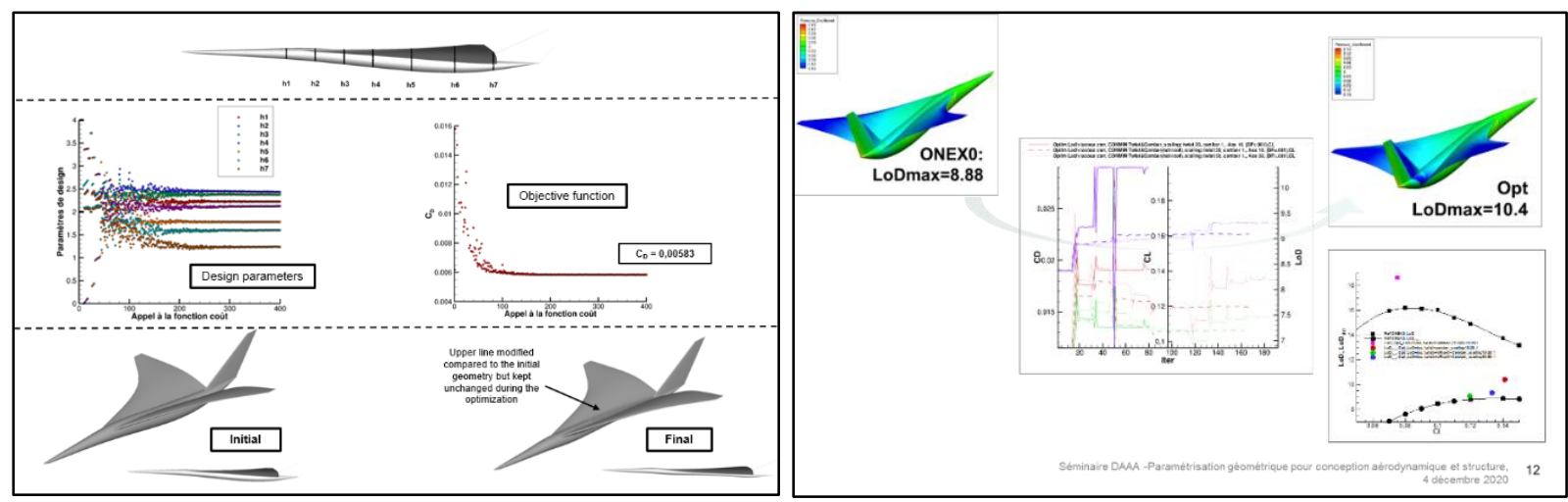

Figure IV-3: First assessment of the capabilities of an aerodynamic/sonic boom MDAO framework for supersonic aero-shape design through fuselage (left) and wing (right) aerodynamic optimizations.

Finally, the capability and flexibility of this approach to deal with the geometrical complexity of a complete aircraft configuration with engine integration has been exercise with success by performing first engine integration design work, as illustrated in Figure IV-4.

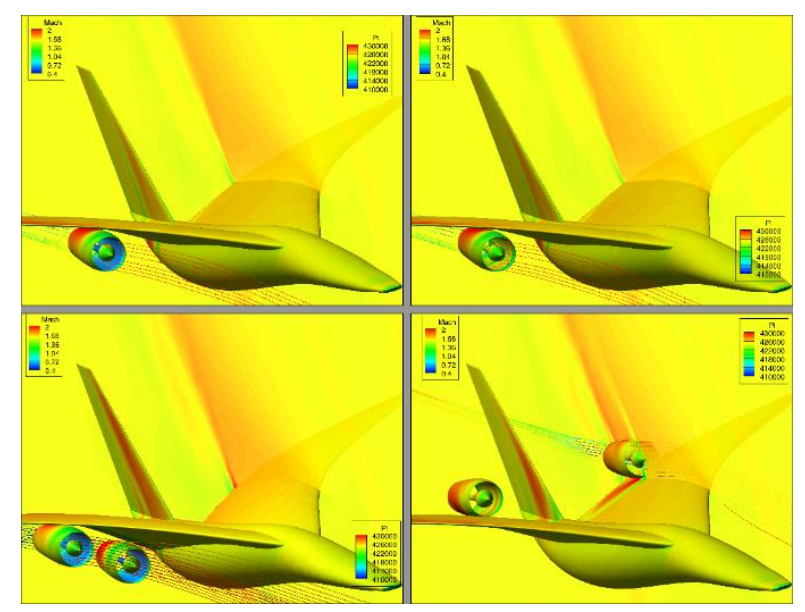

Figure IV-4: Illustration of engine installation investigations to evaluate their effects on aerodynamic performance and sonic boom signatures. 


\section{V.Conclusion}

This paper describes several example of high-fidelity multi-disciplinary simulations-based design frameworks, how they have been set-up at ONERA and applied to multi-disciplinary aircraft design problems. The application of an aero-structural design framework including sensitivity based on the coupled aero-elastic adjoint equations is introduced for the engine pylon design problem of a fully industrially relevant aircraft configuration. Then a bi-objective optimization of both the aerodynamic efficiency and the aeroacoustic noise generated by the leading edge slat of a simplified high-lift system is demonstrated with high fidelity CFD and CAA simulations. Finally, the framework for bi-objective optimization of both aerodynamic drag and sonic boom of a Supersonic Civil Transport aircraft is introduced and preliminary design results are described.

These three different applications involving different multi-physic problems, different geometry parameterizations and mesh deformation or generation methods, and finally different high fidelity solvers, illustrate the spectrum of high-fidelity simulations-based MDO method developed at ONERA.

In the future, several challenges remain to be overcome to extend the applicability of such high-fidelity MDAO systems, including:

- the extension of such capabilities to a wider range of physical models and in particular to propulsion systems and aero-structural-acoustic coupled simulations;

- the validation of such complex multi-disciplinary simulations;

- the deployment of such simulations in industry, considering that it may also raise cultural and organizational challenges.

\section{Acknowledgments}

The MADELEINE project has received funding from the European Union's Horizon 2020 research and innovation program under grant agreement No 769025.

The CRESCENDO was a European Union Horizon 2020 Project funded under the program SC5-012014: Advanced Earth-system models under grant agreement No 641816.

The ARTEMIS project has received funding from the French civil aviation authority (DGAC)

\section{VI.References}

[1] Carrier, G., Mouton, S., Marcelet, M, and Blondeau, C., "Towards aerodynamic design by optimization of transonic transport aircraft in a multi-disciplinary context", 1st CEAS (Council of European Aerospace Societies) European Air and Space Conference, 10 - 13 September, 2007, Berlin, Germany.

[2] Carrier, G., "Multi-disciplinary optimization of a supersonic transport aircraft wing planform", ECCOMAS 2004 conference, Jyväskylä (Finlande), 24-28 July 2004.

[3] Cambier, L., Heib, S., and Plot, S. "The Onera elsA CFD software: input from research and feedback from industry", Mechanics \& Industry 14(3), 159-174 (2013). 
[4] Peter, J., Dwight, R., "Numerical sensitivity analysis for aerodynamic optimization : a survey of approaches." Computers and Fluids, 39 :373-391, 2010

[5] Achard, T., Blondeau, C., Ohayon, R., "High-Fidelity Aerostructural Gradient Computation Techniques with Application to a Realistic Wing Sizing", AIAA Journal, Vol. 56, No. 11, pp. 4488-4499, (2018)

[6] Achard T., " Techniques de calcul de gradient aéro-structure haute-fidélité pour l'optimisation des voilures flexibles", PhD thesis 2017.

[7] L. Giraud, S. Gratton, X. Pinel, and X. Vasseur, Flexible GMRES with deflated restarting, SIAM J. Sci. Comput., 32 (4), pp. 1858-1878, 2010.

[8] Jadoui M., Blondeau C., Martin E., Renac F., Roux F.-X., "Inner-Outer Krylov Solvers for Linear Systems in Structured and High-Order Unstructured CFD Problems“, virtual ECCOMAS 2020, January 2021.

[9] MADELEINE project homepage, https://www.madeleine-project.eu/

[10] Méheut M. et al., "MADELEINE project: challenges of adjoint-based multi-disciplinary optimization in industry", virtual ECCOMAS 2020, January 2021.

[11] Méheut M. et al., "Multidisciplinary Adjoint-based Optimizations in the MADELEINE Project: Overview and Main Results", submitted to AIAA AVIATION 2021.

[12] Mouton, S., Laurenceau, J., Carrier G., "Aerodynamic and Structural Optimization of Powerplant Integration under the Wing of a Transonic Transport Aircraft", $42^{\text {ème }}$ colloque d'Aérodynamique Appliquée AAAF 19-21 mars 2007, Sophia-Antipolis.

[13] CRESCENDO project homepage, https://cordis.europa.eu/project/id/502917/reporting/fr

[14] Salah El Din I., Dumont A. and Blondeau C., "Transonic Wing-body Civil Transport Aircraft Aero-Structural Design Optimization using a Bi-Level High Fidelity Approach - A Focus on the Aerodynamic Process", ASM 2013.

[15] Blondeau C., Salah el Din I., "A Bi-Level High Fidelity Aero-Structural Integrated Design Methodology - A Focus on the Structural Sizing Process", RaeS ASDC 2012.

[16] Sobieszczanski-Sobieski J., Agte J. S., Sandusky R. R., "Bi-level Integrated System Synthesis", AIAA Journal, Vol. 38, No. 1, 2000, pp. 164-172.

[17] Van der Vooren, J., Destarac, D., "Drag / Thrust Analysis of Jet-Propelled Transonic Transport Aircraft: Definition of Physical Drag Components," Aerospace Science and Technology, Vol. 8, No. 7, October 2004.

[18] Bettebghor D., Blondeau C., Toal D. and Eres H., "Bi-objective optimization of pylon-enginenacelle assembly: weight vs. tip clearance criterion", Struct Multidisc Optim 48, 637-652 (2013). https://doi.org/10.1007/s00158-013-0908-7.

[19] Carini M., Blondeau C., Fabbiane N., Méheut M., Abu-Zurayk M., Feldwisch J., Ilic C., Merle A., Olivant R. and Brézillon J., "Towards industrial aero-structural aircraft optimization via coupled-adjoint derivatives", submitted to AIAA AVIATION 2021.

[20] Redonnet, S., Desquesnes, G., Manoha, E., and Parzini, C., "Numerical Study of Acoustic Installation Effects with a Computational Aeroacoustics Method", AIAA Journal, Vol. 48, No. 5, pp. 929-937, (2010).

[21] Goldstein, M. E., “Aeroacoustics”, McGraw-Hill, New York, (1976). 
[22] Bernd Liebhardt, Klaus Luetjens, Volker Gollnick, "Estimation of the Market Potential for Supersonic Airliners via Analysis of the Global Premium Ticket Market", Conference Paper, DOI: $10.2514 / 6.2011-6806$

[23] Rallabhandi, S., Mavris, D., "Aircraft Geometry Design and Optimization for Sonic Boom Reduction", AIAA Journal of Aircraft, Vol. 44, No. 1, pp. 35-47, 2007ONERA CISAP

[24] G. Carrier, "Multi-disciplinary optimization of a supersonic transport aircraft wing planform", Paper presented at ECCOMAS 2004 conference , Jyväskylä (Finlande), 24-28 July 2004

[25] J. Brezillon, G. Carrier, M. Laban, "Multidisciplinary Optimization of Supersonic Aircraft Including Low-Boom Considerations", October 2011, Journal of Mechanical Design 133(10), DOI: $10.1115 / 1.4004972$

[26] A. Minelli, I. Salah El Din, G. Carrier, "Inverse Design Approach for Low-Boom Supersonic Configurations", October 2014, AIAA Journal 52(10):2198-2212, DOI: 10.2514/1.J052834

[27] Harry R. Welge, John Bonet, Todd Magee, Chet Nelson, Eric Adamson, Steve Baughcum, Robert T. Britt, Gregory Miller, Jimmy Tai, "N+2 Supersonic Concept Development and Systems Integration", NASA technical report NASA/CR-2010-216842

[28] John Morgenstern, Michael Buonanno, Jixian Yao, Mugam Murugappan, Umesh Paliath, Lawrence Cheung, Ivan Malcevic, Kishore Ramakrishnan, Nikolai Pastouchenko and Trevor Wood, Steve Martens, Phil Viars, Trevor Tersmette, Jason Lee, Ron Simmons, and David Plybon, Juan Alonso, Francisco Palacios, Trent Lukaczyk, and Gerald Carrier, "Advanced Concept Studies for Supersonic Commercial Transports Entering Service in the 2018-2020 Period Phase 2", NASA technical report NASA/CR—2015-218719

[29] R. Haimes and J. F. Dannenhoffer, "The Engineering Sketch Pad: A Solid-Modeling, FeatureBased, Web-Enabled System for Building Parametric Geometry," in 21st AIAA Computational Fluid Dynamics Conference, 2013.

[30] Steve Karman, Nick Wyman, “Automatic Unstructured Mesh Generation with Geometry Attribution”, AIAA Paper 10.2514/6.2019-1721

[31] Palacios, F., Colonno, M. R., Aranake, A. C., Campos, A., Copeland, S. R., Economon, T. D., Lonkar, A. K., Lukaczyk, T. W., Taylor, T. W. R., and Alonso, J. J., "Stanford University Unstructured (SU2): An Open-source Integrated Computational, Environment for Multi-Physics Simulation and Design", 51st AIAA Aerospace Sciences Meeting, Grapevine, Texas 